\title{
Cumulative nondecoupling effects of Kaluza-Klein neutrinos in electroweak processes
}

\author{
Ara Ioannisian* \\ Max-Planck-Institut für Physik, Föhringer Ring 6, 80805 Munich, Germany \\ Apostolos Pilaftsis \\ Theory Division, CERN, CH-1211 Geneva 23, Switzerland, \\ Fermilab, P.O. Box 500, Batavia, Illinois 60510, \\ and Department of Theoretical Physics, University of Thessaloniki, GR 54006 Thessaloniki, Greece
}

(Received 19 January 2000; published 31 July 2000)

\begin{abstract}
In Kaluza-Klein theories of low-scale quantum gravity, gravitons and isosinglet neutrinos may propagate in a higher-dimensional space with large compact dimensions, whereas all particles of the standard model are confined on a $(1+3)$-dimensional subspace. After compactification of the extra dimensions, the resulting Yukawa couplings of the Kaluza-Klein neutrinos to the lepton doublets become naturally very suppressed by a higher-dimensional volume factor, in agreement with phenomenological observations. We show that oneloop effects induced by Kaluza-Klein neutrinos, albeit tiny individually, act cumulatively in electroweak processes, giving rise to a nondecoupling behavior for large values of the higher-dimensional Yukawa couplings. Owing to the nondecoupling effects of Kaluza-Klein neutrinos, we can derive stronger constraints on the parameters of the theory that originate from the nonobservation of flavor-violating and universalitybreaking phenomena, which involve the $W$ and $Z$ bosons, and the $e, \mu$, and $\tau$ leptons.
\end{abstract}

PACS number(s): $11.25 . \mathrm{Mj}$

\section{INTRODUCTION}

Recently, Arkani-Hamed, Dimopoulos, and Dvali [1] have considered the radical possibility that the fundamental scale of quantum gravity is no longer set by the Planck mass $M_{\mathrm{P}}=1.2 \times 10^{19} \mathrm{GeV}$, but the true scale of quantum gravity $M_{F}$ is many orders of magnitude smaller than $M_{\mathrm{P}}$ close to the $\mathrm{TeV}$ energies. ${ }^{1}$ The observed weakness of gravity is then due to the presence of a number $\delta$ of large extra dimensions, within which only gravity can propagate and, most probably, fields that are singlets under the standard model (SM) gauge group, such as isosinglet neutrinos [7-9]. In this theoretical framework, the SM particles do not feel the presence of the extra dimensions, but are rather confined to a $(1+3)$-dimensional Minkowski subspace that constitutes our observable world. Most interestingly, the ordinary Planck mass $M_{\mathrm{P}}$ is related to the genuinely fundamental scale $M_{F}$ through

\footnotetext{
*On leave of absence from Yerevan Physics Institute, Alikhanian Br. 2, 375036 Yerevan, Armenia.

${ }^{1}$ There have been earlier considerations in the literature that discussed the possibility of lowering the string but not the Planck scale in superstring theories. Most noticeably, Witten [2] and Horava and Witten [3] considered a novel M-theory scenario compactified on an orbifold $S^{1} / Z_{2}$, in which the string scale was lowered by two orders of magnitude to energies of order $10^{16} \mathrm{GeV}$. Along these lines, Lykken [4] discussed an analogous scenario, in which the string scale was further lowered to the $\mathrm{TeV}$ range. In a much earlier work, Antoniadis [5] discussed the possibility of lowering the compactification radius of gauge interactions at the $\mathrm{TeV}$ scale in the context of string theories. In related supersymmetric scenarios, Dienes, Dudas, and Gherghetta [6] have recently studied several aspects of gauge-coupling unification.
}

$$
M_{\mathrm{P}} \approx M_{F}\left(R M_{F}\right)^{\delta / 2},
$$

where $R$ denotes the compactification radii of the extra compact dimensions, which are all taken to be of equal size. The scenario, with $\delta=1$ and $M_{F}$ of order TeV, predicts a visible macroscopic compactification radius and is therefore ruled out. Many recent astrophysical [7-12] and phenomenological [13] studies have been devoted to explore the viability of low-scale theories of quantum gravity, with $\delta \geqslant 2$.

In this paper, we shall study the phenomenological consequences of loop effects of higher-dimensional isosinglet neutrinos on collider and lower energy experiments. Specifically, we find that, both at the tree and quantum levels, higher-dimensional isosinglet neutrinos can naturally induce observable phenomena of lepton-flavor violation and universality breaking in $W$ - and Z-boson interactions. To quantitatively analyze the new-physics effects, we shall consider a minimal higher-dimensional scenario, in which the SM is extended by an isosinglet neutrino $N$ that propagates in 3 $+\delta$ spatial dimensions. The isosinglet neutrino $N$ couples, with nonuniversal Yukawa couplings, to all three lepton SM doublets, $L_{e}, L_{\mu}$, and $L_{\tau}$, which are localized on our threedimensional world. After compactification, the resulting Yukawa couplings of the Kaluza-Klein (KK) neutrinos to the SM leptons come out to be highly suppressed by the volume factor of the extra dimensions $M_{F} / M_{\mathrm{P}} \sim 10^{-16}[7,8]$.

One might now think that the new-physics phenomena mediated by the KK neutrinos would also be extremely suppressed by the same volume factor of the extra dimensions. However, this is not true. After summing over the tower of the KK neutrinos, we obtain an effective theory whose Yukawa interactions are mediated by order-unity Yukawa couplings of the original Lagrangian before compactification. As a consequence, we expect a cumulative nondecoupling phenomenon of the KK neutrinos to occur in loops for large 
higher-dimensional Yukawa couplings: namely, the KK neutrinos appear to violate the known decoupling theorem due to Appelquist and Carazzone [14]. ${ }^{2}$ The higher-dimensional nondecoupling phenomenon is analogous to the one studied in Refs. [15-20], for singlet-neutrino scenarios [21,22] with large $\mathrm{SU}(2)_{L}$ Dirac masses and mixings. Because of the nondecoupling effects of heavy (KK) neutrinos, phenomena of new physics can be dramatically enhanced to an observable level, such as lepton-flavor-violating decays of the $Z$ boson [15], universality-breaking effects in the diagonal leptonic decays of the $Z$ boson [16], neutrinoless two- and three-body decays of the $\tau$ and $\mu$ leptons [17,18], and universalitybreaking effects in leptonic asymmetries measured on the $Z$ pole [19]. In fact, the nonobservation of the above newphysics effects places stringent bounds on the parameter space of the theory. Here, we shall perform an analogous study for the higher-dimensional singlet-neutrino scenario under consideration. The limits obtained by our analysis are rather generic and can easily carry over to related higherdimensional models.

Another important feature of the singlet-neutrino models is the decoupling property of a very high isosinglet mass [23]. In higher-dimensional models, the fundamental Planck mass $M_{F}$ is the one that is now playing the role of the isosinglet mass scale. Thus, we expect that the KK neutrinos decouple from the loops as $M_{F} \rightarrow \infty$. In this limit, all newphysics phenomena mentioned above will be suppressed by inverse powers of $M_{F}$. However, for relatively small values of $M_{F}$, e.g., $M_{F} \leq 100 \mathrm{TeV}$, the screening effect of the higher-dimensional Planck scale will be less dramatic, and experimental information is then needed to place a lower bound on $M_{F}$.

The organization of the paper is as follows. In Sec. II, we describe the basic low-energy structure of a minimal model with one higher-dimensional isosinglet neutrino. In Sec. III, we derive constraints on the parameters of the KK theory, which arise from tree-level contributions to electroweak observables. In Sec. IV, we explicitly demonstrate the cumulative nondecoupling effect of $\mathrm{KK}$ neutrinos in a typical flavor-changing neutral-current (FCNC) graph. In Sec. V, we present analytic results of the loop contributions of the KK neutrinos to electroweak observables of new physics, and also set new limits on the parameters of the theory. Section VI summarizes our conclusions.

\section{HIGHER-DIMENSIONAL MODEL WITH ONE SINGLET NEUTRINO}

For our phenomenological study, we shall adopt a variant [9] of the model discussed in Ref. [7]. Nevertheless, the results of our analysis will equally well apply to other recently

\footnotetext{
${ }^{2}$ This theorem is not directly applicable to spontaneous-symmetrybreaking theories, such as the one we are considering here. The reason is that not all operators of dimension 2 can be increased independently of those of dimension 3 , since they are related by the Higgs mechanism.
}

proposed scenarios [8]. For definiteness, we will be considering a model that minimally extends the SM-field content by one singlet Dirac neutrino $N(x, y)$ which propagates in a $[1+(3+\delta)]$-dimensional Minkowski space. We denote by $x^{\mu}$, with $\mu=0,1,2,3$, the one time and the three spatial coordinates of our observable world and by $y^{k}$, with $k=1, \ldots, \delta$, the new large compact dimensions. The $y$-coordinates are compactified on a circle of radius $R$ by applying the periodic identification $y \equiv y+2 \pi R$. Furthermore, we consider that the higher-dimensional Dirac neutrino $N(x, y)$ generally has nonuniversal Yukawa couplings $\bar{h}_{l}$ to the three ordinary lepton isodoublets $L_{l}(x)$, with $l=e, \mu, \tau$.

For the purpose of illustration, we shall consider that the higher-dimensional Dirac neutrino $N(x, y)$ feels the presence of only one large compact dimension. Then, our results can easily be generalized to higher dimensions. The leptonic sector of the minimal model consists of the following fields:

$$
L_{l}(x)=\left(\begin{array}{c}
\nu_{l L}(x) \\
l_{L}(x)
\end{array}\right), \quad l_{R}(x), \quad N(x, y)=\left(\begin{array}{c}
\xi(x, y) \\
\bar{\eta}(x, y)
\end{array}\right),
$$

where $\nu_{l L}, l_{L}$, and $l_{R}$ describe four-dimensional Weyl spinors of the charged leptons and their associate left-handed neutrinos, and $\xi$ and $\eta$ are two-component spinors in five dimensions. The five-dimensional gamma matrices may be represented by

$$
\gamma_{\mu}=\left(\begin{array}{cc}
0 & \bar{\sigma}_{\mu} \\
\sigma_{\mu} & 0
\end{array}\right) \quad \text { and } \gamma_{4}=\left(\begin{array}{cc}
i \mathbf{1}_{2} & 0 \\
0 & -i \mathbf{1}_{2}
\end{array}\right) \text {, }
$$

where $\sigma^{\mu}=\left(\mathbf{1}_{2}, \vec{\sigma}\right)$ and $\bar{\sigma}^{\mu}=\left(\mathbf{1}_{2},-\overrightarrow{\boldsymbol{\sigma}}\right)$, and $\sigma_{1}, \sigma_{2}$ and $\sigma_{3}$ are the usual Pauli matrices. The effective Lagrangian of our minimal model reads

$$
\begin{aligned}
\mathcal{L}_{\text {eff }}= & \int_{0}^{2 \pi R} d y\left[\bar{N}\left(i \gamma^{\mu} \partial_{\mu}+i \gamma_{4} \partial_{y}\right) N-m \bar{N} N+\delta(y-a)\right. \\
& \left.\times\left(\sum_{l=e, \mu, \tau} \bar{h}_{l} L_{l} \Phi \xi+\text { H.c. }\right)+\delta(y-a) \mathcal{L}_{\mathrm{SM}}(\Phi)\right]
\end{aligned}
$$

where $\widetilde{\Phi}=i \sigma_{2} \Phi^{*}$ and $\mathcal{L}_{\mathrm{SM}}(\Phi)$ describes the SM Lagrangian. The dimensionful Yukawa couplings $\bar{h}_{l}$ may be related to the dimensionless ones $h_{l}$ through

$$
\bar{h}_{l}=\frac{h_{l}}{\left(M_{F}\right)^{\delta / 2}},
$$

with $\delta=1$. Without any further restriction on the parameters of the theory, the reduced couplings $h_{l}$ are expected to be of order unity, as $M_{F}$ is the only available energy scale to normalize the dimensionful couplings $\bar{h}_{l}$. In Eq. (2.3), we have included the bare Dirac bilinear $m \bar{N} N$. As we will see below, 
the effect of this term is to shift the mass of the lowest-lying KK state by an amount $m$. In principle, we could also have added another Lorentz- and gauge-invariant fermionic bilinear in Eq. (2.3), i.e., $M N^{T} C^{(5)-1} N$, where $C^{(5)}=-\gamma_{1} \gamma_{3}$. The presence of the latter operator is not very essential for our phenomenological discussion. In fact, this last term drops out, if one imposes invariance of the Lagrangian (2.3) under a global transformation that respects lepton number $N \rightarrow e^{i \theta} N, L_{l} \rightarrow e^{-i \theta} L_{l}$, and $l_{R} \rightarrow e^{i \theta} l_{R}$.

We can now express the five-dimensional two-component spinors $\xi$ and $\eta$ of $N(x, y)$ in terms of a Fourier series expansion as follows:

$$
\begin{aligned}
& \xi(x, y)=\frac{1}{\sqrt{2 \pi R}} \sum_{n=-\infty}^{\infty} \xi_{n}(x) \exp \left(\frac{i n y}{R}\right), \\
& \eta(x, y)=\frac{1}{\sqrt{2 \pi R}} \sum_{n=-\infty}^{\infty} \eta_{n}(x) \exp \left(\frac{i n y}{R}\right) .
\end{aligned}
$$

Substituting Eqs. (2.5) and (2.6) into the effective Lagrangian (2.3) and then performing the $y$ integration yields

$$
\begin{aligned}
\mathcal{L}_{\mathrm{eff}}= & \mathcal{L}_{\mathrm{SM}}(\Phi)+\sum_{n=-\infty}^{\infty}\left\{\bar{\xi}_{n}\left(i \bar{\sigma}^{\mu} \partial_{\mu}\right) \xi_{n}+\bar{\eta}_{n}\left(i \bar{\sigma}^{\mu} \partial_{\mu}\right) \eta_{n}\right. \\
& -\left[\left(m+\frac{i n}{R}\right) \xi_{n} \eta_{-n}+\text { H.c. }\right] \\
& \left.+\left(\sum_{l=e, \mu, \tau} \bar{h}_{l}^{(n)} L_{l} \Phi \xi_{n}+\text { H.c. }\right)\right\}
\end{aligned}
$$

where

$$
\bar{h}_{l}^{(n)}=\frac{M_{F}}{M_{\mathrm{P}}} h_{l} \exp \left(\frac{i n a}{R}\right) .
$$

As was first noticed in Refs. [7,8], the four-dimensional Yukawa couplings $\bar{h}_{l}^{(n)}$ are naturally suppressed by the volume factor $M_{F} / M_{\mathrm{P}}$ of the extra dimensions.

After spontaneous symmetry breaking (SSB), the effective Lagrangian of the KK neutrino-mass matrix reads

$$
\mathcal{L}_{\text {mass }}^{\mathrm{KK}}=\Psi_{+}^{T} \mathcal{M} \Psi_{-}+\text {H.c. },
$$

where $\quad \Psi_{+}^{T}=\left(\nu_{l L}, \eta_{0}, \eta_{1}, \eta_{-1}, \ldots, \eta_{n}, \eta_{-n}, \ldots\right), \quad \Psi_{-}^{T}$ $=\left(\xi_{0}, \xi_{-1}, \xi_{1}, \ldots, \xi_{-n}, \xi_{n}, \ldots\right)($ with $n>0)$, and

$$
\mathcal{M}=\left(\begin{array}{ccccccc}
m_{l}^{(0)} & m_{l}^{(-1)} & m_{l}^{(1)} & \ldots & m_{l}^{(-n)} & m_{l}^{(n)} & \ldots \\
m & 0 & 0 & \ldots & 0 & 0 & \ldots \\
0 & m-\frac{i}{R} & 0 & \ldots & 0 & 0 & \ldots \\
0 & 0 & m+\frac{i}{R} & \ldots & 0 & 0 & \ldots \\
\vdots & \vdots & \vdots & \ddots & \vdots & \vdots & \ldots \\
0 & 0 & 0 & \ldots & m-\frac{i n}{R} & 0 & \ldots \\
0 & 0 & 0 & \ldots & 0 & m+\frac{i n}{R} & \ldots \\
\vdots & \vdots & \vdots & \vdots & \vdots & \vdots & \ddots
\end{array}\right),
$$

$$
U^{\nu}=\left(\begin{array}{cc}
\left(\mathbf{1}_{3}+\Xi^{*} \Xi^{T}\right)^{-1 / 2} & \Xi^{*}\left(\mathbf{1}+\Xi^{T} \Xi^{*}\right)^{-1 / 2} \\
-\Xi^{T}\left(\mathbf{1}_{3}+\Xi^{*} \Xi^{T}\right)^{-1 / 2} & \left(\mathbf{1}+\Xi^{T} \Xi^{*}\right)^{-1 / 2}
\end{array}\right),
$$
tional lectively denoted as $\nu_{l}$, and can be treated independently of the rectangular part of the neutrino-mass matrix. The massless Weyl spinors $\nu_{l}$ are predominantly left-handed and hence describe the observable neutrinos.

To make this very last point explicit, we will first go from the weak basis $\Psi_{+}$to another rotated basis, e.g., $\Psi_{+}^{R}$, which is defined by the unitary transformation $\Psi_{+}=U^{\nu} \Psi_{+}^{R}$, where $\left(\Psi_{+}^{R}\right)^{T}=\left(\nu_{l}, \eta_{0}^{R}, \eta_{1}^{R}, \eta_{-1}^{R}, \ldots, \eta_{n}^{R}, \eta_{-n}^{R}, \ldots\right)$ and with

$$
\Xi=\left(\frac{m_{l}^{(0)}}{m}, \frac{m_{l}^{(-1)}}{m-\frac{i}{R}}, \frac{m_{l}^{(1)}}{m+\frac{i}{R}}, \ldots, \frac{m_{l}^{(-n)}}{m-\frac{i n}{R}}, \frac{m_{l}^{(n)}}{m+\frac{i n}{R}}, \ldots\right),
$$


and $\Xi^{*}\left(\mathbf{1}+\Xi^{T} \Xi^{*}\right)^{-1 / 2}=\left(\mathbf{1}_{3}+\Xi^{*} \Xi^{T}\right)^{-1 / 2} \Xi^{*}$. In Eq. (2.11), the root of a Hermitian matrix, e.g., $H$ $=\left(\mathbf{1}+\Xi^{T} \Xi^{*}\right)$, is defined as $H^{1 / 2}=U_{H} \hat{H}^{1 / 2} U_{H}^{\dagger}$, where $U_{H}$ is the unitary matrix that diagonalizes $H$, i.e., $\hat{H}=U_{H}^{\dagger} H U_{H}$. It is then easy to verify that $H^{1 / 2} H^{1 / 2}=H$, as it should be. In the newly introduced weak basis, the three upper rows of the rotated neutrino-mass matrix $\mathcal{M}^{R}=\left(U^{\nu}\right)^{T} \mathcal{M}$ vanish identically, giving rise to three massless chiral fields $\nu_{l}$, while the remainder of the matrix assumes the usual rectangular form that describes massive Dirac fields. In fact, for the case at hand, one has $m_{l}^{(n)} \ll m$ and the massless chiral fields $\nu_{l}$ are predominantly left-handed, i.e.,

$$
\nu_{l L}=\left(\mathbf{1}_{3}+\Xi^{*} \Xi^{T}\right)^{-1 / 2}\left(\nu_{l}+\Xi * \Psi_{+}^{\prime R}\right),
$$

with $\nu_{l(L)}^{T}=\left(\nu_{e(L)}, \nu_{\mu(L)}, \nu_{\tau(L)}\right) \quad$ and $\quad\left(\Psi_{+}^{\prime R}\right)^{T}$ $=\left(\eta_{0}^{R}, \eta_{1}^{R}, \eta_{-1}^{R}, \ldots, \eta_{n}^{R}, \eta_{-n}^{R}, \ldots\right)$. In the limit $m \rightarrow 0$ discussed in Ref. [7], there is a level-crossing effect and $\eta_{0}$ becomes massless, whereas one linear combination of the three $\nu_{l}$ fields acquires a small Dirac mass of order $m_{l}^{(0)}$; the other two linear combinations orthogonal to the last one remain massless. The rectangular part of the matrix $\mathcal{M}, \mathcal{M}_{\chi}$, which are spanned by the field vectors $\Psi_{+}^{\prime R}$ and $\Psi_{-}$, can be diagonalized independently by a biunitary transformation $V_{+}^{T} \mathcal{M}_{\chi} V_{-}=\hat{\mathcal{M}}_{\chi}$. We denote the resulting KK mass eigenfields by $\chi^{(n)}$. To leading order in $m_{l}^{(n)} / m$, the mass eigenvalues of $\mathcal{M}_{\chi}$ are given by

$$
\begin{gathered}
\hat{\mathcal{M}}_{\chi} \approx \operatorname{diag}\left(m, \sqrt{m^{2}+\frac{1}{R^{2}}}, \sqrt{m^{2}+\frac{1}{R^{2}}}, \ldots,\right. \\
\left.\sqrt{m^{2}+\frac{n^{2}}{R^{2}}}, \sqrt{m^{2}+\frac{n^{2}}{R^{2}}}, \ldots\right) .
\end{gathered}
$$

In this approximation, up to phase factors, the unitary matrices $V_{ \pm}$stay close to unity [12]. Note that unlike $\chi^{(0)}$, all other massive Dirac fields fall into degenerate pairs, i.e. $m_{(n)}=m_{(-n)}$, with $n>0$. As we have mentioned above, since the chiral neutrino $\nu_{l}$ is massless, the next-to-lightest state of the neutrino mass spectrum $\chi^{(0)}$ exhibits a mass gap of order $m$, i.e., $m_{(0)} \approx m$.

In the following, we shall give the Lagrangians [24] that govern the interactions of the neutrinos $\nu_{l}$ and $\chi^{(n)}$ and the charged leptons $l$ with the gauge bosons $W^{ \pm}$and $Z$, as well as with their respective would-be Goldstone bosons $G^{ \pm}$and $G^{0}$. The interaction Lagrangians can be summarized as follows:

$$
\begin{aligned}
\mathcal{L}_{\text {int }}^{W^{ \pm}}= & -\frac{g_{w}}{\sqrt{2}} W^{-\mu} \sum_{l=e, \mu, \tau}\left(B_{l \nu_{l}} \bar{l} \gamma_{\mu} P_{L} \nu_{l}\right. \\
& \left.+\sum_{n=-\infty}^{\infty} B_{l, n} \bar{l} \gamma_{\mu} P_{L} \chi^{(n)}+\text { H.c. }\right),
\end{aligned}
$$

$$
\begin{aligned}
\mathcal{L}_{\mathrm{int}}^{Z}= & -\frac{g_{w}}{2 c_{w}} Z^{\mu}\left[\sum_{l, l^{\prime}=e, \mu, \tau} C_{\nu_{l} \nu_{l^{\prime}}} \bar{\nu}_{l} \gamma_{\mu} P_{L} \nu_{l^{\prime}}\right. \\
& +\left(\sum_{l=e, \mu, \tau} \sum_{n=-\infty}^{\infty} C_{\nu_{l}, n} \bar{\nu}_{l} \gamma_{\mu} P_{L} \chi^{(n)}+\text { H.c. }\right) \\
& \left.+\sum_{n, k=-\infty}^{\infty} C_{n, k} \bar{\chi}^{(n)} \gamma_{\mu} P_{L} \chi^{(k)}\right], \\
\mathcal{L}_{\mathrm{int}}^{G^{ \pm}}= & -\frac{g_{w}}{\sqrt{2} M_{W}} G^{-} \sum_{l=e, \mu, \tau}\left[B_{l \nu_{l}} m_{l} \bar{l} P_{L} \nu_{l}\right. \\
& \left.+\sum_{n=-\infty}^{\infty} B_{l, n} \bar{l}\left(m_{l} P_{L}-m_{(n)} P_{R}\right) \chi^{(n)}+\text { H.c. }\right],
\end{aligned}
$$

$$
\begin{aligned}
\mathcal{L}_{\text {int }}^{G^{0}=} & \frac{i g_{w}}{2 M_{W}} G^{0}\left[\sum_{l=e, \mu, \tau}\left(\sum_{n=-\infty}^{\infty} C_{\nu_{l}, n} m_{(n)} \bar{\nu}_{l} P_{R} \chi^{(n)}+\text { H.c. }\right)\right. \\
& \left.-\sum_{n, k=-\infty}^{\infty} C_{n, k} \bar{\chi}^{(n)}\left(m_{(n)} P_{L}-m_{(k)} P_{R}\right) \chi^{(k)}\right]
\end{aligned}
$$

where $g_{w}$ is the $\mathrm{SU}(2)_{L}$ coupling constant $c_{w}^{2}=1-s_{w}^{2}$ $=M_{W}^{2} / M_{Z}^{2}, P_{L(R)}=\left[1-(+) \gamma_{5}\right] / 2$ are the chirality projection operators, and $m_{l}$ and $m_{(n)}$ indicate the masses of the charged leptons and KK neutrinos, respectively. The matrices $B$ and $C$ appearing in Eqs. (2.15)-(2.17) are defined as

$$
\begin{aligned}
B_{l \nu_{k}} & =\sum_{i=e, \mu, \tau} V_{l i}^{l} U_{i k}^{\nu} \quad(\text { with } k=e, \mu, \tau), \\
B_{l, n} & =\sum_{i=e, \mu, \tau} V_{l i}^{l} U_{i, n}^{\nu}, \\
C_{\nu_{l} \nu_{l^{\prime}}} & =\sum_{k=e, \mu, \tau} B_{k \nu_{l}} B_{k \nu_{l^{\prime}}}^{*}, \quad C_{\nu_{l}, n}=\sum_{k=e, \mu, \tau} B_{k \nu_{l}} B_{k, n}^{*}, \\
C_{n, m} & =\sum_{k=e, \mu, \tau} B_{k, n} B_{k, m}^{*},
\end{aligned}
$$

where $V^{l}$ is a unitary matrix that occurs in the diagonalization of the charged lepton mass matrix. From Eqs. (2.19) and (2.20), it is interesting to observe that the gauge interactions between the $\mathrm{SU}(2)_{L}$ doublets $L_{l}$ and the KK states $\chi^{(n)}$ are suppressed by a factor $m_{l}^{(n)} / m_{(n)}$, while the corresponding couplings to two KK neutrino states $\chi^{(n)}$ and $\chi^{(m)}$ are further suppressed by the ratio $m_{l}^{(n)} m_{l}^{(m)} /\left(m_{(n)} m_{(m)}\right)$.

\section{TREE-LEVEL CONSTRAINTS}

The interaction Lagrangians (2.15) - (2.17) give rise to important phenomena of new physics both at the tree level and beyond. In this section, we will determine the new-physics contributions of KK neutrinos to electroweak observables, which occur at the tree level, and so derive limits on the 
fundamental Planck scale $M_{F}$ and the mixing parameters of the theory.

The most striking feature of the higher-dimensional scenario is the loss of lepton universality in electroweak processes involving $W$ - and Z-boson interactions. However, KK neutrinos may also lead to observable modifications of the muon lifetime, the invisible width of the $Z$ boson, the cross section of the $\nu e$ deep inelastic scatterings, etc. For our analysis, it proves useful to define the mixing parameters, which were first introduced by Langacker and London [25],

$$
\begin{aligned}
\left(s_{L}^{\nu_{l}}\right)^{2} \equiv & 1-\sum_{l^{\prime}=e, \mu, \tau}\left|B_{l \nu_{l^{\prime}}}\right|^{2} \\
= & \sum_{n=-\infty}^{\infty}\left|B_{l, n}\right|^{2} \\
= & {\left[V^{l}\left(\mathbf{1}_{3}+\Xi * \Xi^{T}\right)^{-1 / 2}\left(\Xi^{*} \Xi^{T}\right)\right.} \\
& \left.\times\left(\mathbf{1}_{3}+\Xi^{*} \Xi^{T}\right)^{-1 / 2} V^{l \dagger}\right]_{l l} .
\end{aligned}
$$

In order to evaluate the last equality on the right-hand side (RHS) of Eq. (3.1), we will approximate the sum over the KK states by a higher-dimensional energy integral, which has an upper ultra-violet (UV) cutoff at $M_{F}$, above which string-threshold effects are expected to become more important. To be precise, we make the replacement

$$
\sum_{n=-\infty}^{\infty} \rightarrow S_{\delta} R^{\delta} \int_{0}^{M_{F}} E^{\delta-1} d E
$$

where $S_{\delta}=2 \pi^{\delta / 2} / \Gamma(\delta / 2)$ is the surface area of a $\delta$-dimensional sphere of unit radius. Furthermore, we consider that the charged lepton mass matrix is diagonal and non-negative, i.e., $V^{l}=\mathbf{1}_{3}$. Then, to leading order in $\left(h_{l} v\right) / M_{F}$, we find that $\left(s_{L}^{\nu_{l}}\right)^{2} \approx\left(\Xi^{*} \Xi^{T}\right)_{l l}$, with

$$
\begin{aligned}
\left(\Xi^{*} \Xi^{T}\right)_{l l} & \left.=\left|m_{l}^{(0)}\right|^{2} S_{\delta} R^{\delta} \int_{0}^{M_{F} d E E^{\delta-1}} \frac{m^{2}+E^{2}}{m^{2}}\right) \text { for } \delta=2, \\
& =\left\{\begin{array}{l}
\frac{\pi h_{l}^{2} v^{2}}{M_{F}^{2}} \ln \left(\frac{M_{F}^{2}+m^{2}}{m^{2}}\right) \\
\frac{S_{\delta}}{\delta-2} \frac{h_{l}^{2} v^{2}}{M_{F}^{2}}\left[1+\mathcal{O}\left(m^{2} / M_{F}^{2}\right)\right] \text { for } \delta>2 .
\end{array}\right.
\end{aligned}
$$

Equation (3.3) shows that deviations of the $W l \nu$ and $Z \nu \nu$ couplings from their SM values are logarithmically enhanced in a theory with two compact dimensions [26]. In the limit $m \rightarrow 0$, in which the scenario of Ref. [7] is recovered, one must simply replace the logarithm $\ln \left[\left(M_{F}^{2}+m^{2}\right) / m^{2}\right]$ by $\ln \left(M_{\mathrm{P}}^{2} / M_{F}^{2}\right)$ in Eq. (3.3). Furthermore, we find that the parameters $\left(s_{L}^{\nu_{l}}\right)^{2}$ are not suppressed by the volume factor $M_{F}^{2} / M_{P}^{2}$, as would have been the case if we had not summed over all the KK states [26]. In fact, after summation over the KK states, we obtain an effective theory in which the
Yukawa interactions are mediated by couplings $h_{l}$ of order unity. As we will see in Secs. IV and V, the latter gives rise to observable nondecoupling effects at the one-loop electroweak order, and can lead to more severe limits on the parameters of the theory than those considered here.

The mixing parameters $\left(s_{L}^{\nu_{l}}\right)^{2}$ may now be constrained by a number of experimental results, which are obtained from (i) the precise measurement of the muon width $\Gamma(\mu$ $\rightarrow e \nu \nu$ ), (ii) the neutrino counting at the $Z$ peak, (iii) charged-current universality in pion decays, i.e., $\Gamma(\pi$ $\rightarrow e \nu) / \Gamma(\pi \rightarrow \mu \nu)$, (iv) charged-current universality in tau decays, i.e., $B(\tau \rightarrow e \nu \nu) / B(\tau \rightarrow \mu \nu \nu)$. In the following, we shall discuss in more detail the constraints obtained from limits on the new-physics phenomena mentioned above.

(i) Precise measurement of the muon lifetime. The presence of KK neutrinos leads to a modification of the muon width $\Gamma(\mu \rightarrow e \nu \nu)$, when compared to the SM result $\Gamma_{\mathrm{SM}}(\mu \rightarrow e \nu \nu)$. In particular, we have

$$
1-\frac{\Gamma(\mu \rightarrow e \nu \nu)}{\Gamma_{\mathrm{SM}}(\mu \rightarrow e \nu \nu)}=\left(s_{L}^{\nu_{e}}\right)^{2}+\left(s_{L}^{\nu_{\mu}}\right)^{2} \lesssim 0.01 .
$$

The upper limit derived above is very conservative, in the sense that we also estimated the impact of neglecting highorder terms. The major new-physics contributions come from one-loop corrections to the $W$-boson propagator. These one-loop corrections are quantified by the electroweak oblique parameters, such as $S, T$, and $U$ [27], and will be discussed in Sec. V. Furthermore, one-loop vertex effects generically introduce further corrections to the mixing parameters $\left(s_{L}^{\nu_{l}}\right)^{2}$, which could be of order $15 \%$ [28].

(ii) Neutrino counting at the $Z$ peak. In the higherdimensional model under study, the coupling of the $Z$ boson to the massless neutrinos $\nu_{l}$ is reduced by a factor 1 $-\left(s_{L}^{\nu_{l}}\right)^{2}$ relative to the SM case. The latter would result in an observable change of the $Z$-boson invisible width at the CERN $e^{+} e^{-}$collider LEP, which is translated into the upper limit [29]

$$
1-\frac{\Gamma(Z \rightarrow \nu \nu)}{\Gamma_{\mathrm{SM}}(Z \rightarrow \nu \nu)}=\left(s_{L}^{\nu_{e}}\right)^{2}+\left(s_{L}^{\nu_{\mu}}\right)^{2}+\left(s_{L}^{\nu_{\tau}}\right)^{2}<0.034
$$

at the $2 \sigma$ level. Equations (3.4) and (3.5) give rather reliable upper limits on the absolute size of $\left(s_{L}^{\nu_{l}}\right)^{2}$. The remaining observables measure possible deviations from lepton universality.

(iii) Charged-current universality in pion decays. One may now define an analogous observable in order to probe $e$ $\mu$ universality, i.e.,

$$
1-\frac{R_{\pi}}{R_{\pi}^{\mathrm{SM}}}=\left(s_{L}^{\nu_{e}}\right)^{2}-\left(s_{L}^{\nu_{\mu}}\right)^{2}
$$

where

$$
R_{\pi}=\frac{\Gamma(\pi \rightarrow e \nu)}{\Gamma(\pi \rightarrow \mu \nu)}
$$


TABLE I. Limits on $M_{F}$ and $h_{l}$ at the $2 \sigma$ level. The case $m=0$ is obtained by replacing $\ln \left(M_{F} / m\right)$ with $\ln \left(M_{\mathrm{P}} / M_{F}\right)$.

\begin{tabular}{|c|c|c|c|c|}
\hline \multirow[b]{2}{*}{ Observable } & \multicolumn{2}{|c|}{$\begin{array}{l}\qquad h_{e}=h_{\mu}=h_{\tau}=h \\
\text { Lower limit on } M_{F} / h[\mathrm{TeV}]\end{array}$} & \multicolumn{2}{|c|}{$\begin{array}{l}\qquad h_{\mu}=0 \text { and } h_{e}=h_{\tau} \\
\text { Lower limit on } M_{F} / h_{\tau}[\mathrm{TeV}]\end{array}$} \\
\hline & $\delta=2$ & $\delta>2$ & $\delta=2$ & $\delta>2$ \\
\hline$\Gamma(\mu \rightarrow e \nu \nu)$ & \multirow{2}{*}{$8.9 \ln ^{1 / 2} \frac{M_{F}}{m}$} & $3.5 S_{\delta}^{1 / 2}$ & \multirow{2}{*}{$6.3 \ln ^{1 / 2} \frac{M_{F}}{m}$} & $2.5 S_{\delta}^{1 / 2}$ \\
\hline$\Gamma_{\mathrm{SM}}(\mu \rightarrow e \nu \nu)$ & & $\sqrt{\sqrt{\delta-2}}$ & & $\sqrt{\delta-2}$ \\
\hline $1-\frac{\Gamma(Z \rightarrow \nu \nu)}{}$ & \multirow{2}{*}{$5.9 \ln ^{1 / 2} \frac{M_{F}}{m}$} & $2.4 S_{\delta}^{1 / 2}$ & \multirow{2}{*}{$4.8 \ln ^{1 / 2} \frac{M_{F}}{m}$} & $1.9 S_{\delta}^{1 / 2}$ \\
\hline$\Gamma_{\mathrm{SM}}(Z \rightarrow \nu \nu)$ & & $\sqrt{\sqrt{\delta-2}}$ & & $\sqrt{\sqrt{\delta-2}}$ \\
\hline \multirow{2}{*}{$1-\frac{R_{\pi}}{R_{\pi}^{\mathrm{SM}}}$} & \multirow[t]{2}{*}{-} & \multirow[t]{2}{*}{-} & \multirow{2}{*}{$18.7 \ln ^{1 / 2} \frac{M_{F}}{m}$} & $7.5 S_{\delta}^{1 / 2}$ \\
\hline & & & & $\sqrt{\delta-2}$ \\
\hline \multirow{2}{*}{$1-\frac{R_{\tau \mu}}{R_{\tau \mu}^{\mathrm{SM}}}$} & - & - & \multirow{2}{*}{$5.7 \ln ^{1 / 2} \frac{M_{F}}{m}$} & $2.3 S_{\delta}^{1 / 2}$ \\
\hline & & & & $\sqrt{\delta-2}$ \\
\hline
\end{tabular}

and $R_{\pi}^{\mathrm{SM}}$ is the $\mathrm{SM}$ result. The experimental analysis of the observable in Eq. (3.6) yields the $2 \sigma$ upper limit [30] $]^{3}$

$$
\left(s_{L}^{\nu_{e}}\right)^{2}-\left(s_{L}^{\nu_{\mu}}\right)^{2}<0.003 \pm 0.006 .
$$

(iv) Charged-current universality in tau decays. Yet, universality-breaking effects in the leptonic sector through charged-current interactions can be examined in the tau decays, $\tau \rightarrow e \nu \nu$ and $\tau \rightarrow \mu \nu \nu$. For this purpose, we first define the quantities

$$
R_{\tau e}=\frac{\Gamma(\tau \rightarrow e \nu \nu)}{\Gamma(\mu \rightarrow e \nu \nu)}, \quad R_{\tau \mu}=\frac{\Gamma(\tau \rightarrow \mu \nu \nu)}{\Gamma(\mu \rightarrow e \nu \nu)} .
$$

By analogy, the non-SM contributions to the above two observables are constrained by [30]

$$
\begin{gathered}
1-\frac{R_{\tau e}}{R_{\tau e}^{\mathrm{SM}}}=\left(s_{L}^{\nu_{\tau}}\right)^{2}-\left(s_{L}^{\nu_{e}}\right)^{2}<0.032 \pm 0.048, \\
1-\frac{R_{\tau \mu}}{R_{\tau \mu}^{\mathrm{SM}}}=\left(s_{L}^{\nu_{\tau}}\right)^{2}-\left(s_{L}^{\nu_{\mu}}\right)^{2}<0.040 \pm 0.048,
\end{gathered}
$$

at the $2 \sigma$ level. More recent experimental analyses of $\tau-e$ and $\tau$ - $\mu$ universality [32] lead to a significant improvement of the above $2 \sigma$ upper limits, i.e.,

\footnotetext{
${ }^{3}$ Most recently, it was noticed [31] that, if $m=0$ in Eq. (2.3), the lower bound on $M_{F}$ that is deduced from charged-current universality in pion decays can be very tight, when phase-space effects of KK states lighter than the pion are taken into account. There are two ways to evade such a limit: (i) one may consider $m \sim M_{W}$ $\gg m_{\pi}$, so the next-to-lightest KK state is much heavier than $\pi^{+}$; (ii) one could assume a kind of $\mu-e$ universality, with $m=0$.
}

$$
\left(s_{L}^{\nu_{\tau}}\right)^{2}-\left(s_{L}^{\nu_{e}}\right)^{2} \quad \text { or }\left(s_{L}^{\nu_{\tau}}\right)^{2}-\left(s_{L}^{\nu_{\mu}}\right)^{2}<0.012
$$

These last limits are competitive with those obtained from considerations of charged-current universality in pion decays.

In Table I we show the lower limits on the fundamental scale $M_{F}$, as well as the upper limits on the higherdimensional Yukawa couplings $h_{l}$, for two representative scenarios. In the first scenario, we have considered the complete universality of the Yukawa couplings, i.e., $h_{e}=h_{\mu}$ $=h_{\tau}=h$. In the second scenario, we assume that the $\mu$ lepton does not mix with the singlet neutrino $N(x, y)$, and $h_{e}$ $=h_{\tau}$. Of course, we might have considered the converse case, in which electron does not mix with $N(x, y)$ instead of the muon, but the predictions that we would obtain then would not differ much. In both scenarios, we assume that the singlet Dirac mass $m$ is of order $M_{W}$. From Table I, we see that precision measurements on muon lifetime offer the most sensitive test of lepton-flavor universality in the first scenario. For instance, if $h=1$ and $m=100 \mathrm{GeV}(m=0)$, we derive the limits

$$
M_{F} \lesssim 20(65), \quad 12.5,9.9 \mathrm{TeV},
$$

for $\delta=2,3$, and 6 large compact dimensions, respectively. In the second scenario, the best bounds are obtained by looking at deviations from charged-current universality in pion decays. Thus, if we take $h_{e}=h_{\tau}=1$ and $m=100 \mathrm{GeV}$ ( $m$ $=0$ ), the following lower bounds on $M_{F}$ may be derived:

$$
M_{F} \lesssim 45(102), 26,21 \mathrm{TeV},
$$

for $\delta=2,3$, and 6 large compact dimensions, respectively. The very same limits apply to a third possible scenario, with $h_{e}=h_{\mu}=0$ and $h_{\tau}=1$. For comparison, we note that the respective lower limits on $M_{F}$ derived from upper bounds on the invisible Z-boson width [see Eq. (3.5)] in the third scenario are weaker, i.e.,

$$
M_{F} \lesssim 8.2(23), \quad 4.7,3.8 \mathrm{TeV},
$$




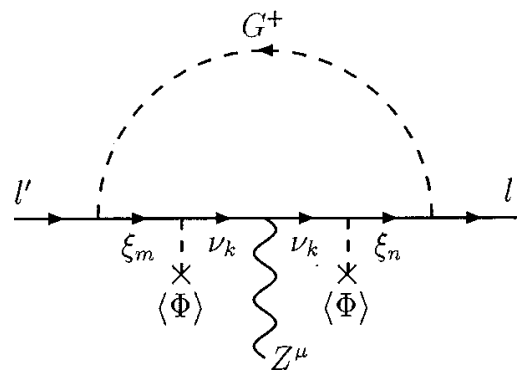

FIG. 1. Feynman graph related to the dominant nondecoupling part of the effective $Z l l^{\prime}$ coupling in the Feynman gauge.

for 2, 3, and 6 large compact dimensions. Notice that the lower limits on $M_{F}$ displayed in Eqs. (3.13)-(3.15) for the three different scenarios increase linearly with the Yukawa couplings $h_{l}$. There may be additional tree-level processes that could constrain the $e-\mu-\tau$ sectors, e.g., observable change of the $\nu e$ deep-inelastic-scattering data. However, the additional constraints turn out to be comparable to those we listed above. In Sec. V, we shall see that one-loop nondecoupling effects of $\mathrm{KK}$ neutrinos can lead to much stronger bounds than those given by Eqs. (3.13)-(3.15).

\section{CUMULATIVE NONDECOUPLING EFFECT OF KALUZA-KLEIN NEUTRINOS}

It is now very instructive to explicitly demonstrate how higher-dimensional Yukawa couplings of order unity give rise to a nondecoupling effect mediated by KK neutrinos in electroweak processes. As an example, we will consider the lepton-flavor-violating vertex $Z l l^{\prime}$, shown in Fig. 1. Similar nondecoupling effects occur in box diagrams involving $\mathrm{KK}$ neutrinos. A more quantitative discussion of one-loop constraints on the parameters of the theory follows in the next section.

Adopting the Feynman gauge for simplicity, the dominant contribution to the lepton-flavor-violating vertex $Z l l^{\prime}$ comes from the Feynman diagram displayed in Fig. 1. The nondecoupling part of the effective $Z l l^{\prime}$ coupling may then be given by

$$
\mathcal{T}\left(Z l l^{\prime}\right)=\sum_{n, m=-\infty}^{\infty} \mathcal{T}_{(n, m)}\left(Z l l^{\prime}\right),
$$

where

$$
\begin{aligned}
\mathcal{T}_{(n, m)}\left(Z l l^{\prime}\right)= & \frac{g_{w}}{4 c_{w}} \bar{h}_{l}^{(n)} \bar{h}_{l^{\prime}}^{(m) *}\left(\sum_{k=e, \mu, \tau} \bar{h}_{k}^{(n) *} \bar{h}_{k}^{(m)}\right) v^{2} \\
& \times \int \frac{d^{4} k}{(2 \pi)^{4} i} P_{R} \frac{1}{k-m_{(n)}} P_{L} \frac{1}{k} \gamma_{\mu} P_{L} \frac{1}{k} \\
& \times P_{R} \frac{1}{k-m_{(m)}} P_{L} \frac{1}{k^{2}-M_{W}^{2}}
\end{aligned}
$$

$$
\begin{gathered}
=\left(\gamma_{\mu} P_{L}\right) \frac{g_{w}}{64 \pi^{2} c_{w}} \frac{M_{F}^{4}}{M_{\mathrm{P}}^{4}} \frac{h_{l} h_{l^{\prime}} v^{2}}{M_{W}^{2}}\left(\sum_{k=e, \mu, \tau} h_{k}^{2}\right) \\
\times \frac{\lambda_{n} \lambda_{m} \ln \left(\lambda_{n} / \lambda_{m}\right)+\lambda_{m} \ln \lambda_{m}-\lambda_{n} \ln \lambda_{n}}{\left(\lambda_{m}-\lambda_{n}\right)\left(1-\lambda_{m}\right)\left(1-\lambda_{n}\right)},
\end{gathered}
$$

with $\lambda_{n}=m_{(n)}^{2} / M_{W}^{2}$. From the last equality in Eq. (4.2), one naively finds that the individual KK contributions $\mathcal{T}_{(n, m)}\left(Z l l^{\prime}\right)$ are indeed tiny, since they are proportional to the volume-dependent suppression factor $M_{F}^{4} / M_{\mathrm{P}}^{4} \approx 10^{-64}$, for $M_{F} \approx 1 \mathrm{TeV}$.

Let us now evaluate the sum over the KK states in Eq. (4.1). The loop function in the last equality of Eq. (4.2) receives its biggest support from KK neutrinos heavier than the $W$ boson. Thus, converting the double sum over the KK states in Eq. (4.1) into a double integral [see Eq. (3.2)] that has an infrared (IR) cutoff at $M_{W} \sim m$, we find

$$
\begin{aligned}
\mathcal{T}\left(Z l l^{\prime}\right) \approx & -\left(\gamma_{\mu} P_{L}\right) \frac{g_{w}}{64 \pi^{2} c_{w}} \frac{M_{F}^{4}}{M_{\mathrm{P}}^{4}} \frac{h_{l} h_{l^{\prime}} v^{2}}{M_{W}^{2}}\left(\sum_{k=e, \mu, \tau} h_{k}^{2}\right) \\
& \times \frac{M_{W}^{2} S_{\delta}^{2}}{4 M_{F}^{2}}\left(M_{F} R\right)^{2 \delta} \int_{m^{2} / M_{F}^{2}}^{1} d z z^{\delta / 2-1} \\
& \times \int_{m^{2} / M_{F}^{2}}^{1} d w w^{\delta / 2-1} \frac{\ln (z / w)}{z-w} .
\end{aligned}
$$

Employing the relation (1.1) that governs the parameters $M_{F}, M_{\mathrm{P}}$, and $R$, we observe that the huge suppression factor $M_{F}^{4} / M_{\mathrm{P}}^{4}$ multiplying the individual $\mathrm{KK}$ contributions is cancelled against the total number of the active KK states $\left(M_{F} R\right)^{2 \delta}$. The double integral in Eq. (4.3) takes on values in the range $1-10^{-2}$, for $2 \leqslant \delta \leqslant 6$. More precisely, to leading order in $\varepsilon \equiv m^{2} / M_{F}^{2} \ll 1$, we have

$$
\begin{aligned}
I_{\delta} & \equiv \frac{1}{4} \int_{\varepsilon}^{1} d z z^{\delta / 2-1} \int_{\varepsilon}^{1} d w w^{\delta / 2-1} \frac{\ln (z / w)}{z-w} \\
& =\left\{\begin{array}{l}
\frac{1}{12} \pi^{2} \quad \text { for } \delta=2, \\
\frac{1}{8} \pi^{2}-1 \quad \text { for } \delta=3, \\
\frac{1}{36} \pi^{2}-\frac{1}{6} \quad \text { for } \delta=4, \\
\frac{1}{16} \pi^{2}-\frac{5}{9} \quad \text { for } \delta=5, \\
\frac{1}{60} \pi^{2}-\frac{1}{8} \text { for } \delta=6 .
\end{array}\right.
\end{aligned}
$$

Then, the effective $Z l l^{\prime}$ coupling may be determined by 


$$
\begin{aligned}
\mathcal{T}\left(Z l l^{\prime}\right) & \approx-\left(\gamma_{\mu} P_{L}\right) \frac{g_{w} \alpha_{w}}{16 \pi c_{w}} \frac{h_{l} h_{l^{\prime}} v^{2}}{M_{F}^{2}}\left(\sum_{k=e, \mu, \tau} \frac{h_{k}^{2} v^{2}}{M_{W}^{2}}\right) S_{\delta}^{2} I_{\delta} \\
& =-\left(\gamma_{\mu} P_{L}\right) \frac{g_{w} \alpha_{w}}{16 \pi c_{w}} s_{L}^{\nu_{l}} s_{L}^{\nu_{l^{\prime}}}\left[\sum_{k=e, \mu, \tau}\left(s_{L}^{\nu_{k}}\right)^{2}\right] \frac{M_{F}^{2}}{M_{W}^{2}} d_{\delta},
\end{aligned}
$$

where $\alpha_{w}=g_{w}^{2} /(4 \pi)$ is the $\mathrm{SU}(2)_{L}$ fine structure constant, $\left(s_{L}^{\nu_{l}}\right)^{2}$ are mixing parameters that have been estimated in Eq. (3.3), and $d_{\delta}$ are dimension-dependent correction factors that take the values

$$
\begin{gathered}
d_{2}=\frac{\pi^{2}}{12 \ln ^{2}\left(M_{F} / m\right)} \approx \frac{0.822}{\ln ^{2}\left(M_{F} / m\right)}, \quad d_{3}=\frac{\pi^{2}}{8}-1 \approx 0.234, \\
d_{4}=\frac{\pi^{2}}{9}-\frac{2}{3} \approx 0.430, \quad d_{5}=\frac{9}{16} \pi^{2}-5 \approx 0.552, \\
d_{6}=\frac{4 \pi^{2}}{15}-2 \approx 0.632 .
\end{gathered}
$$

From Eq. (4.5), we observe that the strength of the effective $Z l l^{\prime}$ coupling increases with the fourth power of the higherdimensional Yukawa couplings $h_{l}$ while it only decreases as $1 / M_{F}^{2}$. Thus, the lower bounds on $M_{F}$, which are derived from limits on new-physics signals mediated by Z-boson interactions, increase quadratically with $h_{l}$.

One might now raise the question whether such a cumulative nondecoupling effect of KK neutrinos could be dramatically weakened if neutrinos would live only in a $\delta$-dimensional subspace of the whole $n$-dimensional space that gravitational interactions would propagate, i.e., $\delta<n$. Nevertheless, it is not difficult to see that this is not true. In this case, the volume factor that suppresses the Yukawa couplings $h_{l}$ is $1 /\left(M_{F} R\right)^{\delta / 2} \approx\left(M_{F} / M_{\mathrm{P}}\right)^{\delta / n}$ [7]. This suppresionvolume factor occuring in the $h_{l}^{4}$-dependent terms will now cancel exactly against the number of the KK-neutrino states in the loop, i.e., $\left(M_{F} R\right)^{2 \delta} \approx\left(M_{\mathrm{P}} / M_{F}\right)^{4 \delta / n}$. Therefore, the analytic results derived in Eqs. (4.5) and (4.6) do not directly depend on the number $n$ of dimensions of the space experienced by gravity. The case $\delta=1$, which was not considered, is not phenomenologically viable, since for the most conservative case with $\delta=1$ and $n=2$, one obtains unacceptably large Yukawa couplings of order $10^{-7}$ for order-unity higher-dimensional Yukawa couplings $h_{l}$, giving rise to neutrino masses in the $\mathrm{keV}$ range.

An analogous cumulative nondecoupling phenomenon, with a kinematic dependence related to the effective $Z l l^{\prime}$ coupling, takes place in box diagrams, which involve $\mathrm{KK}$ modes and two oppositely charged would-be Goldstone bosons $G^{ \pm}$in the loop. Finally, we should remark that the effective $\gamma l l^{\prime}$ vertex considered in Ref. [26] only scales quadratically with the Yukawa coupling $h_{l}$. As we will see in the next section, the bounds on $M_{F}$ derived from the nonobservation of photonic muon decays $\mu \rightarrow e \gamma$ are found to be

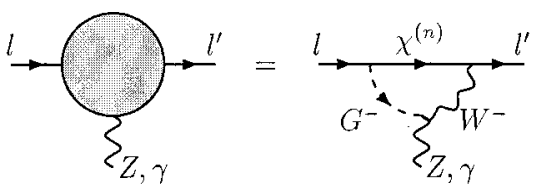

(a)

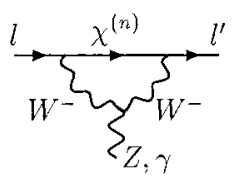

(c)

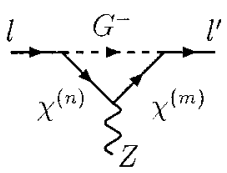

(f)

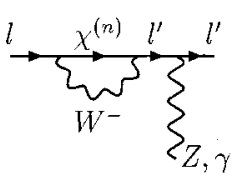

(i)

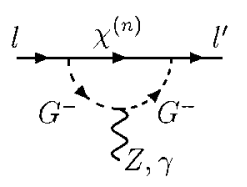

(d)

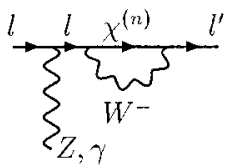

$(\mathrm{g})$

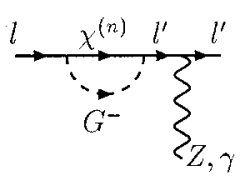

(j)

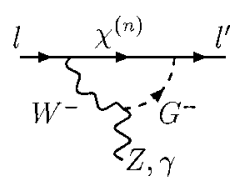

(b)

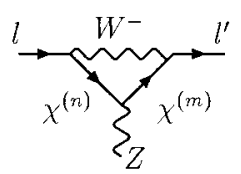

(e)

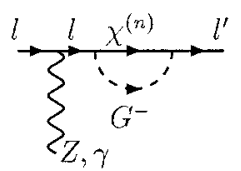

(h)
FIG. 2. Feynman graphs pertaining to the effective $\gamma l l^{\prime}$ and Zll' couplings.

less restrictive than those obtained from $\mu \nmid$ teee and the absence of $\mu$-to-e-conversion events in nuclei.

\section{ONE-LOOP CONSTRAINTS}

After having gained some insight of the cumulative nondecoupling mechanism of KK neutrinos, we shall now present analytic results for the most precisely tested leptonflavor-violating and universality-breaking processes that involve the $W^{ \pm}$and $Z$ bosons, and the $e, \mu$, and $\tau$ leptons. These electroweak processes, which are strictly forbidden in the SM, are induced by $\mathrm{KK}$ states at the one-loop electroweak order. Based on these results, we are then able to derive very stringent limits on the parameters of the theory. Specifically, we confront the predictions of the higherdimensional model with experimental data for the following set of observables of new physics: (i) photonic decays of the muon and tau leptons $\mu \rightarrow e \gamma, \tau \rightarrow e \gamma$, and $\tau \rightarrow \mu \gamma$; (ii) decays of the $\mu$ and $\tau$ leptons into three lighter charged leptons, e.g., $\mu \rightarrow e e e, \tau \rightarrow e e e$, etc.; (iii) coherent $\mu$-to- $e$ conversion in nuclei; (iv) lepton-flavor-violating decays of the $Z$ boson $Z \rightarrow l l^{\prime}$, universality-breaking effects among different diagonal leptonic decays of the $Z$ boson $Z \rightarrow l l$, and among its associate leptonic asymmetries; (v) the electroweak oblique parameters $S, T$, and $U$ [27], and especially $T \propto \delta \rho$ [33].

\section{A. Photonic decays of the $\mu$ and $\tau$ leptons}

As shown in Fig. 2, KK neutrinos can give rise to the photonic FCNC decays $\mu \rightarrow e \gamma$ and $\tau \rightarrow e \gamma$ or $\mu \gamma$. The tran- 
sition element for the generic decay $l\left(p_{l}\right) \rightarrow l^{\prime}\left(p_{l^{\prime}}\right) \gamma(q)$ may conveniently be given by

$$
\begin{aligned}
\mathcal{T}\left(l \rightarrow l^{\prime} \gamma\right)= & \frac{i e \alpha_{w} s_{w}^{2}}{16 \pi M_{W}^{2}} G_{\gamma}^{l l^{\prime}} \varepsilon_{\gamma}^{\mu} \bar{u}_{l^{\prime}} i \sigma_{\mu \nu} q^{\nu} \\
& \times\left[m_{l^{\prime}}\left(1+\gamma_{5}\right)+m_{l}\left(1-\gamma_{5}\right)\right] u_{l},
\end{aligned}
$$

where $G_{\gamma}^{l l^{\prime}}$ is the composite form factor

$$
G_{\gamma}^{l l^{\prime}}=\sum_{n=-\infty}^{\infty} B_{l, n}^{*} B_{l^{\prime}, n} G_{\gamma}\left(\lambda_{n}\right)
$$

with

$$
G_{\gamma}(x)=-\frac{2 x^{3}+5 x^{2}-x}{4(1-x)^{3}}-\frac{3 x^{3} \ln x}{2(1-x)^{4}} .
$$

From this very last equation, it is obvious that the dominant contribution to $G_{\gamma}^{l l^{\prime}}$ comes from values of $\lambda_{(n)} \gg 1$. Then, neglecting terms subleading in $\lambda_{(n)}$ and using the fact that $G_{\gamma}(x)=1 / 2$ in the infinite limit of $x$, the composite form factor $G_{\gamma}^{l l^{\prime}}$ may easily be evaluated to be

$$
G_{\gamma}^{l l^{\prime}}=\frac{1}{2} s_{L}^{\nu_{l}} s_{L}^{\nu_{l^{\prime}}}
$$

where the mixing parameters $s_{L}^{\nu_{l}}$ are defined in Eq. (3.1) and estimated in Eq. (3.3).

Employing Eq. (5.4), the branching ratio of $l \rightarrow l^{\prime} \gamma$ may be determined by

$$
\begin{aligned}
B\left(l \rightarrow l^{\prime} \gamma\right) & =\frac{\alpha_{w}^{3} s_{w}^{2}}{256 \pi^{2}} \frac{m_{l}^{4}}{M_{W}^{4}} \frac{m_{l}}{\Gamma_{l}}\left|G_{\gamma}^{l^{\prime}}\right|^{2} \\
& \approx \frac{\alpha_{w}^{3} s_{w}^{2}}{1024 \pi^{2}} \frac{m_{l}^{4}}{M_{W}^{4}} \frac{m_{l}}{\Gamma_{l}}\left(s_{L}^{\nu_{l}}\right)^{2}\left(s_{L}^{\nu_{l^{\prime}}}\right)^{2} .
\end{aligned}
$$

On the experimental side, we have [29]

$$
\begin{gathered}
B_{\exp }(\mu \rightarrow e \gamma)<4.9(6.0) \times 10^{-11}, \\
B_{\exp }(\tau \rightarrow e \gamma)<2.7(3.3) \times 10^{-6}, \\
B_{\exp }(\tau \rightarrow \mu \gamma)<3.0(3.7) \times 10^{-6},
\end{gathered}
$$

at the $90 \%$ confidence level (C.L.). The numbers in the parentheses refer to upper limits at the $2 \sigma$ level. Only the experimental bound on $B(\mu \rightarrow e \gamma)$ can lead to stronger constraints than those presented in Sec. III. Using the experimentally measured value for the muon width $\Gamma_{\mu}$ $=2.997 \times 10^{-19} \mathrm{GeV}$, we obtain the $2 \sigma$ upper limit on the product $s_{L}^{\nu_{e}} s_{L}^{\nu_{\mu}}$ :

$$
s_{L}^{\nu_{e}} S_{L}^{\nu_{\mu}}<4.5 \times 10^{-4}
$$

(a)

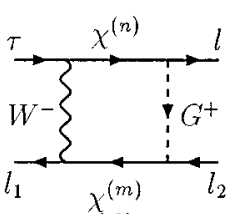

(d)

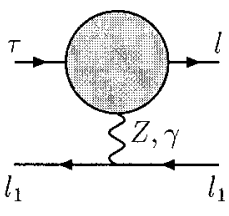

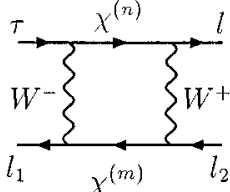

(b)

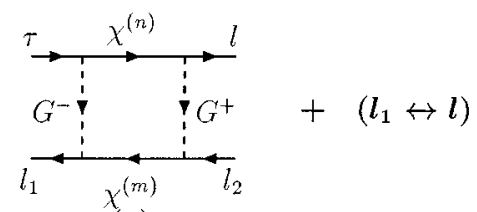

(e)

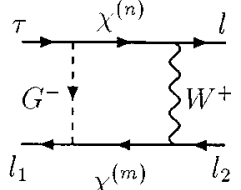

(c)
FIG. 3. Feynman graphs pertaining to the decay $\tau \rightarrow l l_{1} \bar{l}_{2}$.

If we take a scenario with $h_{e}=h_{\mu}=1$ and $m=100 \mathrm{GeV}(\mathrm{m}$ $=0$ ), this very last bound translates into the lower limits on $M_{F}$ :

$$
M_{F} \geq 75(165), 43,33 \mathrm{TeV} \text {, }
$$

for 2, 3, and 6 large compact dimensions, respectively. This result is in qualitative agreement with Ref. [26] for the scenario with $m=0$. In the following, we shall see that, owing to the cumulative nondecoupling effect of KK neutrinos, the non-observation of the decay $\mu \rightarrow e e e$ and the absence of $\mu$-to-e-conversion events in nuclei can lead to a much tighter bound than that given by Eq. (5.8).

\section{B. Neutrinoless three-body decays of the $\mu$ and $\tau$ leptons}

We shall calculate the non-decoupling loop effects of KK neutrinos in three-body decays of the $\mu$ and $\tau$ leptons $\mu$ $\rightarrow e e e, \tau \rightarrow e e e, \tau \rightarrow e \mu \mu, \tau \rightarrow \mu \mu \mu$, and $\tau \rightarrow e e \mu$.

For convenience, we first consider the generic decay process $\tau\left(p_{\tau}\right) \rightarrow l\left(p_{l}\right) l_{1}\left(p_{l_{1}}\right) \bar{l}_{2}\left(p_{l_{2}}\right)$, which is induced by the loop graphs shown in Figs. 2 and 3; analytic expressions for $\mu \rightarrow e e e$ may then be obtained by an obvious interchange of the $\tau$ - $\mu$ kinematic parameters. As can be seen from Fig. 3, there are three amplitudes that contribute to the decay $\tau \rightarrow l l_{1} \bar{l}_{2}$ : the photon-exchange amplitude $\mathcal{T}_{\gamma}$; the $Z$-boson mediated one $\mathcal{T}_{Z}$, and an amplitude describing the box contribution $\mathcal{T}_{\text {box }}$. The three contributing amplitudes are given by

$$
\begin{aligned}
\mathcal{T}_{\gamma}\left(\tau \rightarrow l l_{1} \bar{l}_{2}\right)= & -\frac{i \alpha_{w}^{2} s_{w}^{2}}{4 M_{W}^{2}} \delta_{l_{1} l_{2}} \bar{u}_{l_{1}} \gamma^{\mu} v_{l_{2}} \bar{u}_{l} \\
& \times\left\{F_{\gamma}^{\tau l}\left(\gamma_{\mu}-\frac{q_{\mu} \phi}{q^{2}}\right)\left(1-\gamma_{5}\right)-i G_{\gamma}^{\tau l} \sigma_{\mu \nu}\right. \\
& \left.\times \frac{q^{\nu}}{q^{2}}\left[m_{\tau}\left(1+\gamma_{5}\right)+m_{l}\left(1-\gamma_{5}\right)\right]\right\} u_{\tau},
\end{aligned}
$$




$$
\begin{aligned}
\mathcal{T}_{Z}\left(\tau \rightarrow l l_{1} \bar{l}_{2}\right)= & -\frac{i \alpha_{w}^{2}}{16 M_{W}^{2}} \delta_{l_{1} l_{2}} F_{Z}^{\tau l} \bar{u}_{l} \gamma_{\mu}\left(1-\gamma_{5}\right) \\
& \times u_{\tau} \bar{u}_{l_{1}} \gamma^{\mu}\left(1-4 s_{w}^{2}-\gamma_{5}\right) v_{l_{2}}, \\
\mathcal{T}_{\text {box }}\left(\tau \rightarrow l l_{1} \bar{l}_{2}\right)= & -\frac{i \alpha_{w}^{2}}{16 M_{W}^{2}} F_{\text {box }}^{\tau l l_{1} l_{2}} \bar{u}_{l} \gamma_{\mu}\left(1-\gamma_{5}\right) \\
& \times u_{\tau} \bar{u}_{l_{1}} \gamma^{\mu}\left(1-\gamma_{5}\right) v_{l_{2}},
\end{aligned}
$$

where $q=p_{\tau}-p_{l}$ and [18]

$$
\begin{gathered}
F_{\gamma}^{\tau l}=\sum_{n=-\infty}^{\infty} B_{\tau, n}^{*} B_{l, n} F_{\gamma}\left(\lambda_{n}\right), \\
F_{Z}^{\tau l}=\sum_{n, m=-\infty}^{\infty} B_{\tau, n}^{*} B_{l, m}\left\{\delta_{n m}\left[F_{Z}\left(\lambda_{n}\right)+2 G_{Z}\left(0, \lambda_{n}\right)\right]\right. \\
\left.+C_{n, m}^{*}\left[G_{Z}\left(\lambda_{n}, \lambda_{m}\right)-G_{Z}\left(0, \lambda_{n}\right)-G_{Z}\left(0, \lambda_{m}\right)\right]\right\}, \\
F_{\text {box }}^{\tau l l_{1} l_{2}=} \sum_{n, m=-\infty}^{\infty}\left\{\delta_{n m}\left(B_{\tau, n}^{*} B_{l, m} \delta_{l_{1} l_{2}}+B_{\tau, n}^{*} B_{l_{1}, m} \delta_{l l_{2}}\right)\right. \\
\quad \times\left[F_{\text {box }}\left(0, \lambda_{n}\right)-F_{\text {box }}(0,0)\right]+B_{\tau, n}^{*} B_{l_{2}, m}^{*} \\
\times\left(B_{l, n} B_{l_{1}, m}+B_{l_{1}, n} B_{l, m}\right)\left[F_{\text {box }}\left(\lambda_{n}, \lambda_{m}\right)\right. \\
\left.\left.-F_{\text {box }}\left(0, \lambda_{n}\right)-F_{\text {box }}\left(0, \lambda_{m}\right)+F_{\text {box }}(0,0)\right]\right\} .
\end{gathered}
$$

Note that the composite form factor $G_{\gamma}^{\tau l}$ is defined in Eq. (5.2). In deriving the expressions in Eqs. (5.13) and (5.14), we made use of the unitarity relations, which are obeyed by the $B$ and $C$ matrices defined in Eqs. (2.19) and (2.20). Furthermore, the analytic forms of the loop functions $F_{\gamma}, F_{Z}$, $G_{Z}$, and $F_{\text {box }}$, which may also be found in Refs. [18,34], are given by

$$
\begin{gathered}
F_{\gamma}(x)=\frac{7 x^{3}-x^{2}-12 x}{12(1-x)^{3}}-\frac{\left(x^{4}-10 x^{3}+12 x^{2}\right) \ln x}{6(1-x)^{4}} \\
F_{Z}(x)=-\frac{5 x}{2(1-x)}-\frac{5 x^{2} \ln x}{2\left(1-x^{2}\right)} \\
G_{Z}(x, y)=-\frac{1}{2(x-y)}\left[\frac{x^{2}(1-y) \ln x}{1-x}-\frac{y^{2}(1-x) \ln y}{1-y}\right]
\end{gathered}
$$

$$
\begin{aligned}
F_{\text {box }}(x, y)= & \frac{1}{x-y}\left[( 1 + \frac { x y } { 4 } ) \left(\frac{1}{1-x}+\frac{x^{2} \ln x}{(1-x)^{2}}\right.\right. \\
& \left.-\frac{1}{1-y}-\frac{y^{2} \ln y}{(1-y)^{2}}\right)-2 x y\left(\frac{1}{1-x}+\frac{x \ln x}{(1-x)^{2}}\right. \\
& \left.\left.-\frac{1}{1-y}-\frac{y \ln y}{(1-y)^{2}}\right)\right]
\end{aligned}
$$

As we discussed in Sec. V, to leading order in $M_{F} / M_{W}$, the dominant contributions to the composite form factors in Eqs. (5.12)-(5.14) come from KK states heavier than the $W$ boson. More explicitly, we have

$$
\begin{gathered}
F_{\gamma}^{\tau l} \approx-\frac{7}{12} s_{L}^{\nu_{\tau}} s_{L}^{\nu_{l}}-\frac{c_{\delta}}{6} s_{L}^{\nu_{\tau}} s_{L}^{\nu_{l}} \ln \lambda_{F} \\
F_{Z}^{\tau l} \approx\left(\frac{5}{2}-\frac{3 c_{\delta}}{2} \ln \lambda_{F}\right) s_{L}^{\nu_{\tau}} s_{L}^{\nu_{l}}-\frac{d}{2} s_{L}^{\nu_{\tau}} s_{L}^{\nu_{l}} \sum_{k=e, \mu, \tau}\left(s_{L}^{\nu_{k}}\right)^{2} \lambda_{F}, \\
F_{\text {box }}^{\tau l l_{1} l_{2}} \approx-s_{L}^{\nu_{\tau}} s_{L}^{\nu_{l}} \delta_{l_{1} l_{2}}-s_{L}^{\nu_{\tau}} s_{L}^{\nu_{l_{1}}} \delta_{l l_{2}}+\frac{d_{\delta}}{2} s_{L}^{\nu_{\tau}} s_{L}^{\nu_{l}} s_{L}^{\nu_{l_{1}}} s_{L}^{\nu_{l_{2}}} \lambda_{F},
\end{gathered}
$$

where $\lambda_{F}=M_{F}^{2} / M_{W}^{2}, c_{\delta}=1 / 2$, for $\delta=2$, and $c_{\delta}=1$, for $\delta$ $>2$. In Eqs. (5.20) and (5.21), $d_{\delta}$ are dimension-dependent correction factors, which are given in Eq. (4.6).

The branching ratios for the decays $\tau \rightarrow e \mu \mu$ and $\tau$ $\rightarrow$ eee were calculated in Refs. $[18,17]$. Their analytic expressions may be cast into the form

$$
\begin{aligned}
B(\tau \rightarrow e \mu \mu)= & \frac{\alpha_{w}^{4}}{24576 \pi^{3}} \frac{m_{\tau}^{4}}{M_{W}^{4}} \frac{m_{\tau}}{\Gamma_{\tau}}\left\{\mid F_{\mathrm{box}}^{\tau e \mu \mu}+F_{Z}^{\tau e}\right. \\
& -\left.2 s_{w}^{2}\left(F_{Z}^{\tau e}-F_{\gamma}^{\tau e}\right)\right|^{2}+4 s_{w}^{4}\left|F_{Z}^{\tau e}-F_{\gamma}^{\tau e}\right|^{2} \\
& +8 s_{w}^{2} \operatorname{Re}\left[\left(F_{Z}^{\tau e}+F_{\mathrm{box}}^{\tau e \mu \mu}\right) G_{\gamma}^{\tau e *}\right] \\
& -32 s_{w}^{4} \operatorname{Re}\left[\left(F_{Z}^{\tau e}-F_{\gamma}^{\tau e}\right) G_{\gamma}^{\tau e *}\right] \\
& \left.+32 s_{w}^{4}\left|G_{\gamma}^{\tau e}\right|^{2}\left(\ln \frac{m_{\tau}^{2}}{m_{\mu}^{2}}-3\right)\right\} \\
B(\tau \rightarrow e e e)= & \frac{\alpha_{w}^{4}}{24576 \pi^{3}} \frac{m_{\tau}^{4}}{M_{W}^{4}} \frac{m_{\tau}}{\Gamma_{\tau}}\left\{2 \mid \frac{1}{2} F_{\mathrm{box}}^{\tau e e e}+F_{Z}^{\tau e}\right. \\
& -\left.2 s_{w}^{2}\left(F_{Z}^{\tau e}-F_{\gamma}^{\tau e}\right)\right|^{2}+4 s_{w}^{4}\left|F_{Z}^{\tau e}-F_{\gamma}^{\tau e}\right|^{2} \\
& +16 s_{w}^{2} \operatorname{Re}\left[\left(F_{Z}^{\tau e}+\frac{1}{2} F_{\text {box }}^{\tau e e e}\right) G_{\gamma}^{\tau e *}\right] \\
& -48 s_{w}^{4} \operatorname{Re}\left[\left(F_{Z}^{\tau e}-F_{\gamma}^{\tau e}\right) G_{\gamma}^{\tau e *}\right] \\
& \left.+32 s_{w}^{4}\left|G_{\gamma}^{\tau e}\right|^{2}\left(\ln \frac{m_{\tau}^{2}}{m_{e}^{2}}-\frac{11}{4}\right)\right\} .
\end{aligned}
$$


The branching ratio for the decay $\mu \rightarrow e e e$ may be obtained by making, in Eq. (5.23), the obvious replacements $m_{\tau}$ $\rightarrow m_{\mu}$ and $m_{\mu} \rightarrow m_{e}$. Taking the dominant non-decoupling parts of the composite form factors into account, we obtain for the branching ratios

$$
\begin{aligned}
B(\tau \rightarrow e \mu \mu) \simeq & \frac{\alpha_{w}^{4}}{98304 \pi^{3}} \frac{m_{\tau}^{4}}{M_{W}^{4}} \frac{m_{\tau}}{\Gamma_{\tau}} \frac{M_{F}^{4}}{M_{W}^{4}} d_{\delta}^{2}\left(s_{L}^{\nu_{\tau}}\right)^{2}\left(s_{L}^{\nu_{e}}\right)^{2} \\
& \times\left\{\left(s_{L}^{\nu_{\mu}}\right)^{4}+2\left(1-2 s_{w}^{2}\right)\left(s_{L}^{\nu_{\mu}}\right)^{2}\left[\sum_{l=e, \mu, \tau}\left(s_{L}^{\nu_{l}}\right)^{2}\right]\right. \\
& \left.+8 s_{w}^{4}\left[\sum_{l=e, \mu, \tau}\left(s_{L}^{\nu_{l}}\right)^{2}\right]^{2}\right\}, \\
B(\tau \rightarrow e e e) \simeq & \frac{\alpha_{w}^{4}}{98304 \pi^{3}} \frac{m_{\tau}^{4}}{M_{W}^{4}} \frac{m_{\tau}}{\Gamma_{\tau}} \frac{M_{F}^{4}}{M_{W}^{4}} d_{\delta}^{2}\left(s_{L}^{\nu_{\tau}}\right)^{2}\left(s_{L}^{\nu_{e}}\right)^{2} \\
& \times\left\{\frac{1}{2}\left(s_{L}^{\nu_{e}}\right)^{4}+2\left(1-2 s_{w}^{2}\right)\left(s_{L}^{\nu_{e}}\right)^{2}\left[\sum_{l=e, \mu, \tau}\left(s_{L}^{\nu_{l}}\right)^{2}\right]\right. \\
& \left.+12 s_{w}^{4}\left[\sum_{l=e, \mu, \tau}\left(s_{L}^{\nu_{l}}\right)^{2}\right]^{2}\right\} .
\end{aligned}
$$

The present experimental upper limits on the branching ratios for the lepton-flavor-violating three-body decays of $\mu$ and $\tau$ leptons are [29]

$$
\begin{gathered}
B_{\exp }(\mu \rightarrow e e e)<1.0(1.2) \times 10^{-12}, \\
B_{\exp }(\tau \rightarrow e \mu \mu)<1.8(2.2) \times 10^{-6}, \\
B_{\exp }(\tau \rightarrow e e e)<2.9(3.5) \times 10^{-6}, \\
B_{\exp }(\tau \rightarrow \mu e e)<1.5(1.8) \times 10^{-6}, \\
B_{\exp }(\tau \rightarrow \mu \mu \mu)<1.9(2.3) \times 10^{-6},
\end{gathered}
$$

at the $90 \%$ confidence $(2 \sigma)$ level. In the scenario, with complete Yukawa-coupling universality, i.e., $h_{e}=h_{\mu}=h_{\tau}=h$, the most severe bound on $M_{F}$ comes from $B$ exp $(\mu \rightarrow e e e)$. For $h=1$ and $m=100 \mathrm{GeV}(m=0)$, we obtain the lower limits

$$
M_{F} \gtrsim \begin{cases}250(1000) & \mathrm{TeV} \text { for } \delta=2, \\ 210 \mathrm{TeV} & \text { for } \delta=3-6 .\end{cases}
$$

If the limits in Eq. (5.27) are implemented in this scenario, the predictions obtained for $B(\tau \rightarrow e e e)$ and $B(\tau \rightarrow e \mu \mu)$ are hopelessly small, of order $10^{-12}$, to be tested in any future experiment.

The high upper bounds on $M_{F}$ given in Eqs. (5.8) and (5.27) may be completely avoided in a scenario in which $h_{\mu}=0$ and $h_{e}=h_{\tau}=h$. In this case, $M_{F}$ is only constrained by limits presented in Table I. Implementing these constraints for $h=1$ and $m=100 \mathrm{GeV}(m=0)$ [see also Eq. (3.14)], we find

$$
B(\tau \rightarrow e e e) \leq 7.2 \times 10^{-11}\left(1.4 \times 10^{-10}\right), 6.7 \times 10^{-10},
$$

$$
B(\tau \rightarrow e \mu \mu) \lesssim 5.9 \times 10^{-11}\left(1.2 \times 10^{-10}\right), 6.8 \times 10^{-10} .
$$

The numbers in the parentheses correspond to numerical estimates in a theory with $\delta=2,3-6$ large extra dimensions, respectively. The predictions given in Eq. (5.28) for $B(\tau$ $\rightarrow e \mu \mu)$ and $B(\tau \rightarrow e e e)$ may be enhanced by a factor $10^{3}$ for large Yukawa couplings $h \sim 5$, to the $10^{-7}$ level, which might be probed in future experiments.

\section{Coherent $\boldsymbol{\mu}$-to-e conversion in nuclei}

Coherent $\mu \rightarrow e$ conversion in nuclei, e.g., $\mu^{-}{ }_{22}^{48} \mathrm{Ti}$ $\rightarrow e^{-48} \mathrm{Ti}$, constitutes one of the most sensitive experiments that can set severe bounds on lepton-flavor-violating physics $[35,36]$. The existence of KK neutrinos may give rise to a sizeable $\mu \rightarrow e$ conversion in nuclei, which comes from the following $\gamma-, Z-$, and box-mediated transition amplitudes

$$
\begin{aligned}
\mathcal{T}_{\gamma}(\mu \rightarrow e)= & \frac{i \alpha_{w}^{2} s_{w}^{2}}{4 M_{W}^{2}} \bar{u}_{e}\left[F_{\gamma}^{\mu e} \gamma_{\nu}\left(1-\gamma_{5}\right)-i G_{\gamma}^{\mu e} \sigma_{\nu \lambda}\right. \\
& \left.\times \frac{q^{\lambda}}{q^{2}} m_{\mu}\left(1+\gamma_{5}\right)\right] u_{\mu}\left(\frac{2}{3} \bar{u}_{u} \gamma^{\nu} u_{u}-\frac{1}{3} \bar{u}_{d} \gamma^{\nu} u_{d}\right),
\end{aligned}
$$

$$
\begin{aligned}
\mathcal{T}_{Z}(\mu \rightarrow e)= & \frac{i \alpha_{w}^{2}}{16 M_{W}^{2}} F_{Z}^{\mu e} \bar{u}_{e} \gamma_{\nu}\left(1-\gamma_{5}\right) u_{\mu} \\
& \times\left[\left(1-\frac{8}{3} s_{w}^{2}\right) \bar{u}_{u} \gamma^{\nu} u_{u}-\left(1-\frac{4}{3} s_{w}^{2}\right) \bar{u}_{d} \gamma^{\nu} u_{d}\right] \\
\mathcal{T}_{\mathrm{box}}(\mu \rightarrow e)= & -\frac{i \alpha_{w}^{2}}{16 M_{W}^{2}} \bar{u}_{e} \gamma_{\nu}\left(1-\gamma_{5}\right) u_{\mu} \\
& \times\left[F_{\mathrm{box}}^{\mu e u u} \bar{u}_{u} \gamma^{\nu} u_{u}+F_{\mathrm{box}}^{\mu e d d} \bar{u}_{d} \gamma^{\nu} u_{d}\right]
\end{aligned}
$$

where

$$
\begin{aligned}
F_{\mathrm{box}}^{\mu e u u} & =\sum_{n=-\infty}^{\infty} B_{\mu, n}^{*} B_{e, n}\left[F_{\mathrm{box}}\left(0, \lambda_{n}\right)-F_{\mathrm{box}}(0,0)\right], \\
F_{\mathrm{box}}^{\mu e d d}= & \sum_{n=-\infty}^{\infty}\left\{B_{\mu, n}^{*} B_{e, n}\left[F_{\mathrm{box}}\left(0, \lambda_{n}\right)-F_{\mathrm{box}}(0,0)\right]\right. \\
& +B_{\mu, n}^{*} B_{e, n}\left|V_{t d}\right|^{2}\left[F_{\mathrm{box}}\left(\lambda_{t}, \lambda_{n}\right)-F_{\mathrm{box}}\left(0, \lambda_{n}\right)\right. \\
& \left.\left.-F_{\mathrm{box}}\left(0, \lambda_{t}\right)+F_{\mathrm{box}}(0,0)\right]\right\},
\end{aligned}
$$

with $\lambda_{t}=m_{t}^{2} / M_{W}^{2}$ and $\left|V_{t d}\right| \approx 0.01 \quad$ [29]. In Eqs. (5.29)(5.31), we have only considered the vectorial coupling of the $u$ and $d$ quarks, which is coherently enhanced in nuclei, 
whereas the axial part of the quark couplings describes spindependent interactions, and therefore, their total contribution is almost vanishing [35,36].

Following Refs. [35,36], we consider the kinematic approximations: $q^{2} \approx-m_{\mu}^{2}$ and $p_{e}^{0} \approx\left|\vec{p}_{e}\right| \approx m_{\mu}$, which are applicable for $\mu \rightarrow e$ conversion in nuclei. Then, for nuclei with nucleon numbers $(N, Z)$, we obtain

$$
\begin{aligned}
B_{\mu e}(N, Z) & \equiv \frac{\Gamma[\mu(N, Z) \rightarrow e(N, Z)]}{\Gamma[\mu(N, Z) \rightarrow \text { capture }]} \\
& \approx \frac{\alpha_{\mathrm{em}}^{3} \alpha_{w}^{4} m_{\mu}^{5}}{32 \pi^{2} M_{W}^{4} \Gamma_{\text {capt }}} \frac{Z_{\mathrm{eff}}^{4}}{Z}\left|F\left(-m_{\mu}^{2}\right)\right|^{2}\left|Q_{W}\right|^{2},
\end{aligned}
$$

where $\alpha_{\mathrm{em}}=1 / 137$ is the electromagnetic fine structure constant, $Z_{\text {eff }}$ is the effective atomic number of coherence (e.g., $Z_{\text {eff }} \approx 17.6$ for ${ }_{22}^{48} \mathrm{Ti}$ ) [37], $\left|F\left(-m_{\mu}^{2}\right)\right| \approx 0.54$ is the nuclear form factor [38], and $Q_{W}=V_{u}(2 Z+N)+V_{d}(Z+2 N)$, with

$$
\begin{gathered}
V_{u}=\frac{2}{3} s_{w}^{2}\left(F_{\gamma}^{\mu e}-G_{\gamma}^{\mu e}-F_{Z}^{\mu e}\right)+\frac{1}{4}\left(F_{Z}^{\mu e}-F_{\mathrm{box}}^{\mu e u u}\right), \\
V_{d}=-\frac{1}{3} s_{w}^{2}\left(F_{\gamma}^{\mu e}-G_{\gamma}^{\mu e}-F_{Z}^{\mu e}\right)-\frac{1}{4}\left(F_{Z}^{\mu e}+F_{\mathrm{box}}^{\mu e d d}\right) .
\end{gathered}
$$

The strictest upper limit on $B_{\mu e}(N, Z)$ is obtained from experimental data of $\mu \rightarrow e$ conversion in ${ }_{22}^{48} \mathrm{Ti}$ [39]:

$$
B_{\mu e}^{\exp }(26,22)<4.3(5.24) \times 10^{-12},
$$

at the $90 \%$ confidence $(2 \sigma)$ level. For numerical predictions, we use the experimental value of the muon nuclear capture rate $\Gamma\left[\mu_{22}^{48} \mathrm{Ti} \rightarrow\right.$ capture $] \approx 1.705 \times 10^{-18} \mathrm{GeV}[40]$.

For definiteness, we shall now consider the scenario with all Yukawa couplings equal to unity and $m=100 \mathrm{GeV}(m$ $=0)$. Then, the experimental upper bound on $B_{\mu e}(26),(22)$ gives the following lower limits on $M_{F}$ :

$$
M_{F} \gtrsim \begin{cases}380(1430) & \mathrm{TeV} \text { for } \delta=2, \\ 310 \mathrm{TeV} & \text { for } \delta=3-6 .\end{cases}
$$

These limits represent the most severe bounds that can be derived on the fundamental quantum gravity scale $M_{F}$ from laboratory experiments, for large higher-dimensional Yukawa couplings, e.g., $h_{l} \sim 1$.

\section{Lepton-flavor violation and breaking of universality at the $Z$ peak}

Loop effects of KK neutrinos may induce a number of lepton-flavor and universality-breaking phenomena on the $Z$-boson pole. The most striking experimental signals of new physics would be FCNC Z-boson decays into different leptons, e.g., $Z \rightarrow l l^{\prime}$. Further new-physics signals would be the detection of breaking of universality in the leptonic partial widths $Z \rightarrow l \bar{l}$ and in the $Z$-boson leptonic asymmetries, which may be probed by looking at the observables $[16,19]$

$$
\begin{gathered}
U_{\mathrm{br}}^{\left(l l^{\prime}\right)}=\frac{\Gamma(Z \rightarrow l \bar{l})-\Gamma\left(Z \rightarrow l^{\prime} \bar{l}^{\prime}\right)}{\Gamma(Z \rightarrow l \bar{l})+\Gamma\left(Z \rightarrow l^{\prime} \bar{l}^{\prime}\right)}-U_{\mathrm{br}}^{\mathrm{PS}} \\
=\frac{|\mathcal{T}(Z \rightarrow l \bar{l})|^{2}-\left|\mathcal{T}\left(Z \rightarrow l^{\prime} \bar{l}^{\prime}\right)\right|^{2}}{|\mathcal{T}(Z \rightarrow l \bar{l})|^{2}+\left|\mathcal{T}\left(Z \rightarrow l^{\prime} \bar{l}^{\prime}\right)\right|^{2}}, \\
\Delta \mathcal{A}_{l l^{\prime}}=\frac{\mathcal{A}_{l}-\mathcal{A}_{l^{\prime}}}{\mathcal{A}_{l}+\mathcal{A}_{l^{\prime}}}=\left(\frac{1}{\mathcal{A}_{l}^{\mathrm{SM}}}-1\right) U_{\mathrm{br}}^{\left(l l^{\prime}\right)}, \quad \text { with } l \neq l^{\prime},
\end{gathered}
$$

where $U_{\mathrm{br}}^{\mathrm{PS}}$ indicates known phase-space corrections due to the finiteness of the charged-lepton masses, and $\mathcal{A}_{l}^{\mathrm{SM}}=0.14$ is the SM prediction [29]. In deriving the last equality of Eq. (5.39), we used the theoretical fact that the induced coupling of the $Z$ boson to charged leptons is predominantly left handed, as is the case in our higher-dimensional singletneutrino model [see Eq. (5.10)].

The branching ratio of the FCNC decay $Z \rightarrow l l^{\prime}$ is given by

$$
\begin{aligned}
B\left(Z \rightarrow l \bar{l}^{\prime} \text { or } \bar{l} l^{\prime}\right)= & \frac{\alpha_{w}^{3}}{48 \pi^{2} c_{w}^{2}} \frac{M_{Z}}{\Gamma_{Z}}\left|\mathcal{F}_{Z}^{l l^{\prime}}\left(M_{Z}^{2}\right)\right|^{2} \\
\approx & \frac{\alpha_{w}^{3}}{768 \pi^{2} c_{w}^{2}} \frac{M_{Z}}{\Gamma_{Z}} \frac{M_{F}^{4}}{M_{W}^{4}} d_{\delta}^{2}\left(s_{L}^{\nu_{l}}\right)^{2}\left(s_{L}^{\nu_{l^{\prime}}}\right)^{2} \\
& \times\left[\sum_{k=e, \mu, \tau}\left(s_{L}^{\nu_{k}}\right)^{2}\right]^{2}
\end{aligned}
$$

where the loop function $\mathcal{F}_{Z}^{l l^{\prime}}\left(M_{Z}^{2}\right)$ was calculated in Refs. $[15,18]$. To obtain the last equality in Eq. (5.40), we used the approximation $\mathcal{F}_{Z}^{l l^{\prime}}\left(M_{Z}^{2}\right) \approx \mathcal{F}_{Z}^{l l^{\prime}}(0)=F_{Z}^{l l^{\prime}} / 2$, where $F_{Z}^{l l^{\prime}}$ is given by Eq. (5.13). Furthermore, the universality-breaking observable $U_{\mathrm{br}}^{\left(l l^{\prime}\right)}$ is found to be

$$
\begin{aligned}
U_{\mathrm{br}}^{\left(l l^{\prime}\right)}= & \frac{\alpha_{w}}{2 \pi} \frac{1-2 s_{w}^{2}}{\left(1-2 s_{w}^{2}\right)^{2}+4 s_{w}^{4}}\left[\mathcal{F}_{Z}^{l l}\left(M_{Z}^{2}\right)-\mathcal{F}_{Z}^{l^{\prime} l^{\prime}}\left(M_{Z}^{2}\right)\right] \\
\approx & \frac{\alpha_{w}}{8 \pi} \frac{1-2 s_{w}^{2}}{\left(1-2 s_{w}^{2}\right)^{2}+4 s_{w}^{4}} \frac{M_{F}^{2}}{M_{W}^{2}} d_{\delta}\left[\left(s_{L}^{\nu_{l}}\right)^{2}-\left(s_{L}^{\nu_{l^{\prime}}}\right)^{2}\right] \\
& \times\left[\sum_{k=e, \mu, \tau}\left(s_{L}^{\nu_{k}}\right)^{2}\right] .
\end{aligned}
$$

The current experimental situation on $B\left(Z \rightarrow l l^{\prime}\right)$ and $U_{\mathrm{br}}^{\left(l l^{\prime}\right)}$ is as follows [29]:

$$
\begin{gathered}
B_{\exp }(Z \rightarrow e \mu)<1.7 \times 10^{-6}, \quad B_{\exp }(Z \rightarrow e \tau)<9.8 \times 10^{-6}, \\
B_{\exp }(Z \rightarrow \mu \tau)<1.2 \times 10^{-5},
\end{gathered}
$$

and, almost independently of the lepton flavors $l$ and $l^{\prime}$, 


$$
U_{\mathrm{br}}^{\left(l l^{\prime}\right) \exp }=\frac{1}{2}\left(1-\frac{\Gamma\left(Z \rightarrow l^{\prime} l^{\prime}\right)}{\Gamma(Z \rightarrow l l)}\right)=5.0 \times 10^{-3},
$$

at the $2 \sigma$ level. The experimental limits on the universalitybreaking parameter $\Delta \mathcal{A}_{l l^{\prime}}$ are slightly weaker than those derived by $U_{\mathrm{br}}^{\left(l l^{\prime}\right)}$, if one uses the relation given by Eq. (5.39), i.e., $\Delta \mathcal{A}_{l l},<3.0 \times 10^{-2}$.

In view of the constraints derived in Secs. V A-V C newphysics phenomena in Z-boson decays with electrons and muons only in the final state are far beyond the realm of detection. Therefore, we shall discuss the numerical predictions obtained for the FCNC $Z$-boson decay $Z \rightarrow e \tau$ and $U_{\mathrm{br}}^{(\mu \tau)}$ in a phenomenologically more favorable scenario, with $h_{e}=h_{\tau}=h$ and $h_{\mu}=0$. Considering $h=1$ and $m=100 \mathrm{GeV}$ ( $m=0)$, and the lower limits on $M_{F}$ stated in Eq. (3.14), we obtain the following upper limits for $\delta=2$ and 3-6:

$$
\begin{gathered}
B(Z \rightarrow e \tau) \lesssim 2.9 \times 10^{-10}\left(5.5 \times 10^{-10}\right), \quad 3.8 \times 10^{-9}, \\
U_{\mathrm{br}}^{(\mu \tau)} \lesssim 5.0 \times 10^{-5}\left(8.8 \times 10^{-5}\right), \quad 1.9 \times 10^{-4} .
\end{gathered}
$$

For maximal values of the Yukawa couplings $h \sim 5$ the above predictions for $B(Z \rightarrow e \tau)$ and $U_{\mathrm{br}}^{(\mu \tau)}$ increase by a factor $10^{3}$ and 25 , respectively.

Finally, it is illuminating to discuss the upper bounds that can be obtained for $M_{F}$ in a more weakly constrained scenario, i.e., $h_{e}=h_{\mu}=0$ and $h_{\tau} \neq 0$. In addition, we assume that this scenario is only constrained by experimental limits on the invisible width of the $Z$ boson [cf. see Eq. (3.15)]. If $h_{\tau}$ $=1$ and $m=100 \mathrm{GeV}(m=0)$, the experimental bounds on $U_{\mathrm{br}}^{(e \tau)}$ and $U_{\mathrm{br}}^{(\mu \tau)}$ yield

$$
M_{F} \gtrsim 3(31) \mathrm{TeV}, \quad \text { for } \delta=2-6 \text {. }
$$

Note that these limits are almost comparable to those deduced by experimental data on the invisible $Z$-boson width in Eq. (3.15).

\section{E. Contribution to the electroweak oblique parameters}

Finally, KK neutrinos may manifest their presence by inducing sizeable contributions to the electroweak oblique parameters $S, T$, and $U$ [27]. We find that a cumulative nondecoupling effect of KK states also occurs here, very similar the previous flavor-dependent observables. For the kind of the new physics we are considering, $T$ turns out to be the most sensitive electroweak oblique parameter, which is related to Veltman's $\rho$ parameter [33] through

$$
\rho-1=\alpha_{\mathrm{em}}\left(M_{Z}\right) T=\frac{\Pi_{W W}^{\mathrm{KK}}(0)}{M_{W}^{2}}-\frac{\Pi_{Z Z}^{\mathrm{KK}}(0)}{M_{Z}^{2}} .
$$

In Eq. (5.46), $\Pi_{W W}^{\mathrm{KK}}(0)$ and $\Pi_{Z Z}^{\mathrm{KK}}(0)$ indicate the $\mathrm{KK}$ neutrino contributions to the $W$ - and $Z$-boson self-energies, respectively.

The most recent experimental constraint on $T$ is $T=$ $-0.21 \pm 0.16(+0.10)$, for $M_{H}=M_{Z}(300 \mathrm{GeV})$ [29]. This corresponds to the $2 \sigma$ upper bound on $(\rho-1)$ :

$$
(\rho-1)_{\exp } \leqslant 2.42 \times 10^{-3},
$$

for a Higgs-boson mass $M_{H}=300 \mathrm{GeV}$. On the other hand, to leading order in $M_{F} / M_{W}$, the electroweak oblique parameter $(\rho-1)$ is found to be

$$
\rho-1 \approx \frac{\alpha_{w}}{16 \pi} \frac{M_{F}^{2}}{M_{W}^{2}} d_{\delta}\left[\sum_{l=e, \mu, \tau}\left(s_{L}^{\nu_{l}}\right)^{2}\right]^{2} .
$$

Combining this last theoretical prediction with the limit in Eq. (5.47), we find for $m=100 \mathrm{GeV}(m=0)$

$$
\left(\frac{M_{F}}{1 \mathrm{TeV}}\right) \geqslant\left\{\begin{array}{l}
2.2(25) \times\left(\sum_{l=e, \mu, \tau} h_{l}^{2}\right), \quad \text { for } \delta=2, \\
0.4 d_{\delta}^{1 / 2}\left(\sum_{l=e, \mu, \tau} h_{l}^{2}\right) \frac{S_{\delta}}{\delta-2}, \quad \text { for } \delta>2 .
\end{array}\right.
$$

The above constraints are much weaker than those derived from limits on nonoblique new-physics observables.

Before closing Sec. V, we wish to comment on the fact that there may exist theoretical uncertainties related to the one-loop results. In the loop calculations, it is usually considered that the KK theory is truncated at some scale $M_{F}^{\prime}$ close to $M_{F}$, where $M_{F}^{\prime}$ represents the energy scale of the active KK states, beyond which string effects are still negligible [6]. In the present analysis, we assumed that $M_{F}^{\prime}$ $=M_{F}$. Depending on the dynamics of a given string theory, however, one generally has $M_{F}^{\prime} \leqslant M_{F}$, i.e., $x_{F} \equiv M_{F}^{\prime} / M_{F}$ $\leqslant 1$. Thus, for $x_{F} \neq 1$, the nondecoupling contributions discussed in Sec. IV [e.g., the $Z l l^{\prime}$ coupling given by Eq. (4.5)] must be multiplied by an additional factor $\left(x_{F}\right)^{2 \delta}$. For example, if $x_{F}=0.9$ and $\delta=6$, this additional multiplicative factor is $\sim 0.3$. On the other hand, there may exist compensating factors, which can be obtained by modestly rescaling the normalization of the higher-dimensional Yukawa couplings $h_{l}$ in Eq. (2.4). For instance, if $h_{l}$ is rescaled by a factor 1.35 for $\delta=6$, then the factor $\left(x_{F}\right)^{2 \delta}$, with $x_{F}=0.9$, drops out completely. Because of the above theoretical uncertainties, which are generally inherent in all truncated KK theories, the numerical predictions for the new-physics phenomena and the derived limits should rather be viewed as order-of-magnitude estimates.

\section{CONCLUSIONS}

We studied the phenomenological implications of KaluzaKlein theories of low-scale quantum gravity, which may naturally predict, in addition to gravitons, the presence of singlet neutrinos. In these theories, the singlet neutrinos can propagate in a higher-dimensional space, which is endowed with a number $\delta$ of large compact spatial dimensions. Such low-scale KK theories that include higher-dimensional singlet neutrinos are very appealing, since they can naturally provide suppression mechanisms for understanding the smallness in mass of the observed light neutrinos [7,8], explain the observed anomalies in solar and atmospheric neu- 
trino data through neutrino oscillations $[8,12]$, and finally account for the baryon asymmetry in the Universe through leptogenesis [9].

Another very interesting feature of KK theories of lowscale quantum gravity is that the presence of KK-neutrino states can give rise to a number of testable new-physics signals at collider and lower energies, such as lepton-flavor violation in muon, tau, and Z-boson decays, coherent $\mu \rightarrow e$ conversion in nuclei, and effects of universality breaking in $Z$ and $W$-boson interactions. We confronted the predictions of a minimal higher-dimensional singlet-neutrino model for the aforementioned new-physics phenomena with current experimental data. We found that KK neutrinos heavier than the $W$ boson act cumulatively in the loops, thereby leading to a nondecoupling phenomenon, for large values of the original higher-dimensional Yukawa couplings of the theory. Because of this cumulative nondecoupling phenomenon of the KK states, we were able to derive very stringent constraints on lepton-flavor violation in the $\mu-e$ sector, which originate from upper limits on $\mu \rightarrow e$ conversion in nuclei and $\mu$ teeee. In fact, the limits derived in this way can be much stronger than those obtained from $\mu \nmid e \gamma$ by an earlier consideration [26], for higher-dimensional Yukawa couplings of order unity. Specifically, for $h_{e}=h_{\mu}=h_{\tau}=1$, we derived the rather tight lower limits: $M_{F} \geqslant 380$ (1430) and $310 \mathrm{TeV}$, for 2 and 3-6 large extra dimensions, respectively, and $m=100$ $\mathrm{GeV}(m=0)$. As was illustrated at the end of Sec. V, however, we should stress again that these lower limits are quite generic and must only be considered as order-of-magnitude estimates due to inherent theoretical uncertainties of the string dynamics at the fundamental quantum-gravity scale $M_{F}$.

New-physics effects in $e-\tau$ and $\mu-\tau$ sectors are less constrained than those found for the $e-\mu$ one. In general, one may avoid most of the latter constraints by setting the higher-dimensional Yukawa couplings $h_{e}$ or $h_{\mu}$ to zero. However, even if $h_{e}$ and $h_{\mu}$ are vanishingly small, the nonobservation of universality breaking in leptonic Z-boson decays, which involve $\tau$ leptons in the final state, together with experimental data on the invisible $Z$-boson width are sufficient to place a lower bound on $M_{F}$. For $h_{\tau}=1$ and $h_{\mu}$ $=h_{e}=0$, the lower limit on $M_{F}$ was found to be $M_{F} \geqslant 3$ $\mathrm{TeV}$, almost independently of the number of large compact dimensions. Even though the analysis was performed in a minimal higher-dimensional model, these last bounds, as well as those pertaining to the $e-\mu$ sector, are generically rather model-independent, as long as order-unity Yukawa couplings, $h_{l}$, are present in the original theory before compactification. In this context, it is important to emphasize again that all of the above "one-loop" lower limits on $M_{F}$ scale quadratically with $h_{l}$, for $h_{l} \geqslant 1$. This is an important consequence of the cumulative nondecoupling effect that results from summing up KK states heavier than the electroweak scale. For instance, if $h_{l} \sim 3$, the lower bounds on $M_{F}$ will then increase by one order of magnitude. On the other hand, for maximal values of $h_{l} \sim 5$, the branching ratios for $\tau \rightarrow e \mu \mu$ and $Z \rightarrow e \tau$ could reach the $10^{-7}$ and $10^{-6}$ levels, respectively. Next-generation colliders have the potential capabilities to probe these predictions of KK theories of low-scale quantum gravity and to impose new constraints on their parameters.

\section{ACKNOWLEDGMENTS}

We wish to thank Nima Arkani-Hamed and Savas Dimopoulos for discussions. A.P. thanks the Theory Groups of SLAC and Fermilab for their kind hospitality, while part of this work was done.
[1] N. Arkani-Hamed, S. Dimopoulos, and G. Dvali, Phys. Lett. B 429, 263 (1998); I. Antoniadis, N. Arkani-Hamed, S. Dimopoulos, and G. Dvali, ibid. 436, 257 (1998).

[2] E. Witten, Nucl. Phys. B471, 135 (1996).

[3] P. Hořava and E. Witten, Nucl. Phys. B460, 506 (1996); B475, 94 (1996).

[4] J.D. Lykken, Phys. Rev. D 54, 3693 (1996).

[5] I. Antoniadis, Phys. Lett. B 246, 377 (1990); I. Antoniadis, K. Benakli, and M. Quiros, ibid. B331, 313 (1994).

[6] K.R. Dienes, E. Dudas, and T. Gherghetta, Phys. Lett. B 436, 55 (1998); Nucl. Phys. B537, 47 (1999).

[7] N. Arkani-Hamed, S. Dimopoulos, G. Dvali, and J. MarchRussell, hep-ph/9811448.

[8] K.R. Dienes, E. Dudas, and T. Gherghetta, Nucl. Phys. B557, 25 (1999).

[9] A. Pilaftsis, Phys. Rev. D 60, 105023 (1999).

[10] N. Arkani-Hamed, S. Dimopoulos, and G. Dvali, Phys. Rev. D 59, 086004 (1999).

[11] N. Kaloper and A. Linde, Phys. Rev. D 59, 101303 (1999); R. Sundrum, ibid. 59, 085010 (1999); N. Arkani-Hamed, S. Dimopoulos, and J. March-Russell, ibid. (to be published), hep-th/9809124; D.H. Lyth, Phys. Lett. B 448, 191 (1999); G. Dvali and S.H. Henry Tye, ibid. 450, 72 (1999); T. Banks, M. Dine, and A. Nelson, J. High Energy Phys. 06, 019 (1999); C. Csaki, M. Graesser, and J. Terning, Phys. Lett. B 456, 16 (1999); A. Riotto, Phys. Rev. D 61, 123506 (2000); J.M. Cline, ibid. 61, 023513 (2000); N. Arkani-Hamed, S. Dimopoulos, N. Kaloper, and J. March-Russell, Nucl. Phys. B567, 189 (2000); S. Cullen and M. Perelstein, Phys. Rev. Lett. 83, 268 (1999); L.J. Hall and D. Smith, Phys. Rev. D 60, 085008 (1999); G. Dvali and G. Gabadadze, Phys. Lett. B 460, 47 (1999); G. Dvali, ibid. 459, 489 (1999); P. Binétruy, C. Deffayet, and D. Langlois, Nucl. Phys. B565, 269 (2000); E. Halyo, ibid. B561, 17 (1999); Phys. Lett. B 461, 109 (1999); J. High Energy Phys. 09, 012 (1999); C. Csaki, M. Graesser, C. Kolda, and J. Terning, Phys. Lett. B 462, 34 (1999); D.J.H. Chung and K. Freese, Phys. Rev. D 61, 023511 (2000); A. Campbell-Smith, J. Ellis, N.E. Mavromatos, and D.V. Nanopoulos, Phys. Lett. B 466, 11 (1999).

[12] G. Dvali and A. Yu. Smirnov, Nucl. Phys. B563, 63 (1999).

[13] G.F. Giudice, R. Rattazzi, and J.D. Wells, Nucl. Phys. B544, 3 (1999); T. Han, J.D. Jykken, and R.-J. Zhang, Phys. Rev. D 59, 
105006 (1999); E.A. Mirabelli, M. Perelstein, and M.E. Peskin, Phys. Rev. Lett. 82, 2236 (1999); J.L. Hewett, ibid. 82, 4765 (1999); S. Nussinov and R. Shrock, Phys. Rev. D 59, 105002 (1999); C.P. Burgess, L.E. Ibañez, and F. Quevedo, Phys. Lett. B 447, 257 (1999); T.G. Rizzo, Phys. Rev. D 59, 115010 (1999); 60, 115010 (1999); L. Randall and R. Sundrum, Nucl. Phys. B557, 79 (1999); Phys. Rev. Lett. 83, 3370 (1999); S.A. Abel and S.F. King, Phys. Rev. D 59, 095010 (1999); T. Kobayashi, J. Kubo, M. Mondragon, and G. Zoupanos, Nucl. Phys. B550, 99 (1999); K. Agashe and N.G. Deshpande, Phys. Lett. B 456, 60 (1999); M.L. Graesser, Phys. Rev. D 61, 074019 (2000); P. Nath and M. Yamaguchi, ibid. 60, 116004 (1999); 60, 116006 (1999); M. Masip and A. Pomarol, ibid. 60, 096005 (1999); K. Cheung and W.-Y. Keung, ibid. 60, 112003 (1999); N. Arkani-Hamed and M. Schmaltz, ibid. 61, 033005 (2000); P. Mathews, S. Raychaudhuri, and K. Sridhar, hep-ph/9904232; Phys. Lett. B 461, 196 (1999); H. Davoudiasl, Phys. Rev. D 60, 084022 (1999); 61, 044018 (2000); A. Kehagias and K. Sfetsos, Phys. Lett. B 472, 39 (2000); T.G. Rizzo and J.D. Wells, Phys. Rev. D 61, 016007 (2000); Z.K. Silagadze, hep-ph/9907328; R. Casalbuoni, S. De Curtis, D. Dominici, and R. Gatto, Phys. Lett. B 462, 48 (1999).

[14] T. Appelquist and J. Carazzone, Phys. Rev. D 11, 2856 (1975).

[15] J.G. Körner, A. Pilaftsis, and K. Schilcher, Phys. Lett. B 300, 381 (1993).

[16] J. Bernabéu, J.G. Körner, A. Pilaftsis, and K. Schilcher, Phys. Rev. Lett. 71, 2695 (1993).

[17] A. Pilaftsis, Mod. Phys. Lett. A 9, 3595 (1994); M.C. Gonzalez-Garcia and J.W.F. Valle, ibid. 7, 477 (1992); Mod. Phys. Lett. E 9, 2569 (1994).

[18] A. Ilakovac and A. Pilaftsis, Nucl. Phys. B437, 491 (1995).

[19] J. Bernabéu and A. Pilaftsis, Phys. Lett. B 351, 235 (1995).

[20] For related works, see, G. Bhattacharya, P. Kalyniak, and I. Mello, Phys. Rev. D 51, 3569 (1995); A. Pilaftsis, ibid. 52, 459 (1995); A. Ilakovac, B.A. Kniehl, and A. Pilaftsis, ibid. 52, 3993 (1995); A. Ilakovac, ibid. 54, 5653 (1996); M. Frank and H. Hamidian, ibid. 54, 6790 (1996); P. Kalyniak and I. Mello, ibid. 55, 1453 (1997); G. Barenboim and M. Raidal, Nucl. Phys. B484, 63 (1997); Z. Gagyi-Palffy, A. Pilaftsis, and K. Schilcher, ibid. B513, 517 (1998); S. Fajfer and A. Ilakovac, Phys. Rev. D 57, 4219 (1998); M. Raidal and A. Santamaria, Phys. Lett. B 421, 250 (1998); M. Czakon, M. Zralek, and J. Gluza, hep-ph/9906356.

[21] D. Wyler and L. Wolfenstein, Nucl. Phys. B218, 205 (1983); E. Witten, ibid. B268, 79 (1986); R.N. Mohapatra and J.W.F.
Valle, Phys. Rev. D 34, 1642 (1986); S. Nandi and U. Sarkar, Phys. Rev. Lett. 56, 564 (1986); A.S. Joshipura and U. Sarkar, ibid. 57, 33 (1986).

[22] J. Bernabéu, A. Santamaria, J. Vidal, A. Mendez, and J.W.F. Valle, Phys. Lett. B 187, 303 (1987).

[23] G. Senjanović and A. Sokorac, Nucl. Phys. B164, 305 (1980).

[24] J. Schechter and J.W.F. Valle, Phys. Rev. D 22, 2227 (1980); for notation presented here, see, A. Pilaftsis, Z. Phys. C 55, 275 (1992).

[25] P. Langacker and D. London, Phys. Rev. D 38, 886 (1988).

[26] A.E. Faraggi and M. Pospelov, Phys. Lett. B 458, 237 (1999).

[27] M.E. Peskin and T. Takeuchi, Phys. Rev. Lett. 65, 964 (1990); Phys. Rev. D 46, 381 (1992); G. Altarelli and R. Barbieri, Phys. Lett. B 253, 161 (1991).

[28] B.A. Kniehl and A. Pilaftsis, Nucl. Phys. B474, 286 (1996).

[29] Particle Data Group, C. Caso et al., Eur. Phys. J. C 3, 1 (1998).

[30] D.A. Bryman, Comments Nucl. Part. Phys. 21, 101 (1993), and references therein.

[31] A. Das and O.C.W. Kong, Phys. Lett. B 470, 149 (1999).

[32] For instance, see, A. Pich, hep-ph/9701263, and references therein.

[33] M. Veltman, Nucl. Phys. B123, 89 (1977); Acta Phys. Pol. B 8, 475 (1977).

[34] T. Inami and C.S. Lim, Prog. Theor. Phys. 65, 297 (1981); 65, 1772 (1981); E. Ma and A. Pramudita, Phys. Rev. D 22, 214 (1980); M. Gronau, C. Leung, and J. Rosner, ibid. 29, 2539 (1984).

[35] G. Feinberg and S. Weinberg, Phys. Rev. Lett. 3, 111 (1959); 3, 244 (1959); W.J. Marciano and A.I. Sanda, ibid. 78, 1512 (1977); O. Shanker, Phys. Rev. D 20, 1608 (1979); J. Bernabéu, E. Nardi, and D. Tommasini, Nucl. Phys. B409, 69 (1993).

[36] For reviews, see, J.D. Vergados, Phys. Rep. 133, 1 (1986); T.S. Kosmas, G.K. Leontaris, and J.D. Vergados, Prog. Part. Nucl. Phys. 33, 397 (1994).

[37] J.C. Sens, Phys. Rev. 113, 679 (1959); K.W. Ford and J.G. Wills, Nucl. Phys. 35, 295 (1962); R. Pla and J. Bernabéu, An. Fis. 67, 455 (1971); H.C. Chiang, E. Oset, T.S. Kosmas, A. Faessler, and J.D. Vergados, Nucl. Phys. A559, 526 (1993).

[38] For instance, see, B. Frois and C.N. Papanicolas, Annu. Rev. Nucl. Sci. 37, 133 (1987), and references therein.

[39] C. Dohmen et al., SINDRUM II Collaboration, Phys. Lett. B 317, 631 (1993).

[40] T. Suzuki, D.F. Measday, and J.P. Roalsvig, Phys. Rev. C 35, $2212(1987)$ 